\title{
Poincaré surfaces of section around a 3D irregular body: the case of asteroid 4179 Toutatis
}

\author{
G. Borderes-Motta ${ }^{\star}$ and O. C. Winter ${ }^{\star}$ \\ Grupo de Dinâmica Orbital e Planetologia, São Paulo State University, 12516-410 Guaratinguetá, São Paulo, Brazil
}

Accepted 2017 November 8. Received 2017 October 5; in original form 2017 September 2

\begin{abstract}
In general, small bodies of the Solar system, e.g. asteroids and comets, have a very irregular shape. This feature affects significantly the gravitational potential around these irregular bodies, which hinders dynamical studies. The Poincaré surface of section technique is often used to look for stable and chaotic regions in two-dimensional dynamic cases. In this work, we show that this tool can be useful for exploring the surroundings of irregular bodies such as the asteroid 4179 Toutatis. Considering a rotating system with a particle, under the effect of the gravitational field computed three dimensionally, we define a plane in the phase space to build the Poincare surface of section. Despite the extra dimension, the sections created allow us to find trajectories and classify their stabilities. Thus, we have also been able to map stable and chaotic regions, as well as to find correlations between those regions and the contribution of the third dimension of the system to the trajectory dynamics as well. As examples, we show details of periodic (resonant or not) and quasi-periodic trajectories.
\end{abstract}

Key words: methods: numerical-celestial mechanics-minor planets, asteroids: individual: Toutatis.

\section{INTRODUCTION}

Many bodies of the Solar system do not have enough mass to be in hydrostatic equilibrium. Such condition allows the bodies to have an irregular and asymmetrical shape, as it happens with comets and asteroids. These astronomical objects arouse the interest of the scientific and civil community due to information they provide about the beginning of the Solar system, the danger that they may represent to Earth (Shustov, Naroenkov \& Efremova 2017) and the mining missions that can be performed in these bodies (Abell et al. 2017). Besides this growing interest in those astronomical bodies, the number of space missions to those bodies are growing as well. Some examples of those space missions are shown as follow.

NASA's mission NEAR-Schoemaker (Prockter et al. 2002), JAXA's Hayabusa (Kawaguchi, Fujiwara \& Uesugi 2008) and ESA's ROSETTA (Roll, Witte \& Arnold 2016) visited asteroids 433 Eros, 25143 Itokawa, and comet 67P/Churyumov-Gerasimenko, respectively, and they are examples of successful missions sent to irregular bodies. Currently, the missions JAXA's Hayabusa-2 (Kawaguchi et al. 2008) and NASA's OSIRES-Rex (Kawaguchi et al. 2008) aim to explore the asteroids 1621731999 JU3 and 101995 Bennu, respectively. Some daring manoeuvres were performed in some of the missions quoted and will be repeated in the others. NEAR-Schoemaker spacecraft was the first one to touch the

^E-mail: gabriel_borderes@yahoo.com.br (GB-M); ocwinter@gmail.com $(\mathrm{OCW})$ surface of a small body (433 Eros). The Hayabusa spacecraft accomplished a touch-down on the surface of 25143 Itokawa. During this manoeuvre, the spacecraft collected material from the surface of the asteroid, which was sent to Earth (Kawaguchi et al. 2008). Hayabusa-2 (Kawaguchi et al. 2008) and OSIRES-Rex (Lauretta et al. 2017) intend to collect material from their respective asteroids. Hayabusa-2 (Kawaguchi et al. 2008) and ROSETTA (Roll et al. 2016) even use modules for studies in situ.

For the missions to be successful, the spacecrafts must orbit the targets and collect data using their tools, as mentioned previously. For this reason, accurate studies on orbital dynamics around irregular bodies are needed. Therefore, many works have been made enriching the knowledge concerning the structure of gravitational potential of irregular bodies. Frouard \& Compère (2012) used two numerical models to study the short and long-term evolution of the asteroid system 87 Sylvia, they showed the deeply stable zone, fast and secular chaotic regions of the system. Araujo et al. (2012) used $N$-body simulations and calculated stable regions around triple asteroid 2001 SN263. They found the size and the location of the stabilities zones within the system. Jiang, Baoyin \& Li (2015b) used the periodic orbits to study the stable region in the potential field of the primary body of triple asteroid 216 Kleopatra. In this work, we show the usefulness of one of those methods that allows us to explore regions around irregular and asymmetric bodies and determine the size and location of the stable and unstable regions.

In order to explore the dynamical region around an irregularly shaped body, we adopt the Poincaré surface of section technique. It brings the possibility of identifying the location and size of the regular and chaotic regions, as well as providing information about 
resonances, periodic orbits, and quasi-periodic orbits. This technique has been widely and successfully applied to orbital dynamics. More than half century ago, a significant number of numerical studies of the restricted three-body problem via Poincaré surface of section started (Hénon 1965a,b, 1966a, b, 1969; Jefferys 1971). A more comprehensive research for a specific mass ratio followed those studies a couple of decades ago (Winter \& Murray 1994a,b). Using the restricted three-body problem for a Sun-Jupiter system, Winter \& Murray (1997a,b) analysed first-order resonances and libration regions. Winter (2000) applied the Poincare surface of section technique to the Earth-Moon system, where stability regions and periodic orbits were found, and the maximum libration amplitude of the quasi-periodic orbits around a family of periodic orbits was computed. In all the mentioned works, the Poincaré surface of section technique was applied to the two-dimensional (2D) problem, the planar, circular restricted three-body problem.

However, we are interested in applying the technique to irregular and non-symmetric bodies, a 3D problem. Scheeres et al. (1996) used the Poincaré map in order to find a periodic orbit around the asteroid 4769 Castália, whose gravitational potential is irregular and 3D. In the context of the application of Poincaré surface of section technique to a single body, Broucke \& Elipe (2005) considered a circular ring to model a gravitational potential and find several families of periodic orbits, as well as dynamic structures around the ring. Since the ring is a planar object, the problem is also planar. Another planar case was used in Silva, Winter \& Prado (2009). The authors analysed the region around planar shapes (a square and a triangular plate) via Poincaré surface of section and determined stable and unstable regions. Najid, Haj Elourabi \& Zegoumou (2011) used the Poincaré surface of section around an inhomogeneous straight segment. Such segment was set out perpendicular to the rotating system defined. The gravitational potential generated by this segment is $2 \mathrm{D}$, since the non-homogenity of the segment density is a function that makes the gravitational potential symmetric. Liu, Baoyin \& Ma (2011) used the polyhedron model to compute the gravitational potential of a $3 \mathrm{D}$ cube, thus, enabling the mapping by the Poincare surface of section at the cube vicinity and periodic orbits around the cube were found. All the mentioned works treat $2 \mathrm{D}$ or $3 \mathrm{D}$ cases. However, whenever it was a $3 \mathrm{D}$ case, it had symmetry, which made it turn into a 2D case as well. Differently from then, Scheeres et al. (1996) took into account the third dimension in a non-symmetric system, focusing only on determining periodic orbits. Stable and unstable regions were not explored at all.

In order to broadly and systematically explore the stable and chaotic regions around irregular and asymmetric bodies, we use the Poincaré surface of section technique. Our aim is to show that, even with more degrees of freedom, the known structures of Poincaré surfaces of sections are not in general destroyed. In this way, the technique is not restricted to look for periodic orbits, but also to allow the mapping of all the dynamic structures around the body.

In the next two sections, we introduce the main tools used in this work; the mascons model and the Poincare surface of section technique. In Section 4, we present and discuss the results for a study about the asteroid 4179 Toutatis. Stable regions, periodic orbits, quasi-periodic orbits and chaotic regions around this asteroid are identified and analysed. In the last section, the final comments and general observations are presented.

\section{SHAPE MODEL: MASCONS}

The computation of gravitational potential of irregular and small bodies composes the foundation for a dynamical study around these bodies. This physical amount can be represented as an expansion by harmonic series for any body (MacMillan 1936). The gravitational potential is better understood and represented, in terms of accuracy, when its harmonic series have a high number of gravitational coefficients. Another method to evaluate the gravitational field, via the dimensions of the body, is given by Ivory's approach, which consists of fitting a triaxial ellipsoid whose dimensions resemble the body (Laplace 1846; Kellogg 1954).

The polyhedron shape model, a tool that has been developed to model irregular bodies, has been helping to compute a gravitational potential closer to a real one. It provides, with a high level of accuracy, the shape of an observed body (Neese 2004). The shape given by this model is usually a polyhedron of triangular faces that fits the real irregular surface of the body. In order to simplify the computational procedures, it is recommended to decompose the polyhedron into other geometric shapes, like tetrahedrons. With these tetrahedrons, at least two approaches are possible. The first approach is called polyhedron model, and it was developed by Werner (1994). It is based on a volumetric integration of tetrahedrons in order to obtain the gravitational potential. The other approach was first applied in Geissler et al. (1996) and then called 'mascons' model (mass concentration) (Werner \& Scheeres 1996; Rossi, Marzari \& Farinella 1999). Such model is based on filling the polyhedron volume with massive points in order to reproduce the mass distribution of the body. There are, at least, two approaches to distribute these points inside the polyhedron volume. In the first, the points are uniformly distributed in a 3D space (Geissler et al. 1996). In the second, the points are systematically set by using the geometric features of the tetrahedrons (Venditti 2013).

In this work, we adopted the mascons model (Geissler et al. 1996) whose main advantage is the control that can be exerted on the accuracy and the computational speed. The number of mascons is directly proportional to the accuracy and inversely proportional to the integration speed.

Our mascon model was built starting from a uniform grid of points. This grid has dimensions larger than the original polyhedron (Geissler et al. 1996) and is centred at the geometric centre of the polyhedron. The points of the grid that do not belong to the volume of the polyhedron are excluded. The limit of the grid is the polyhedron itself, i.e. the grid has the irregular shape of the body. The gravitational potential of the body, which allows to integrate orbits, to evaluate the equilibrium points, and to study other dynamical features, is computed via the contribution of the sum of the massive points.

\section{POINCARÉ SURFACE OF SECTION TECHNIQUE}

Having defined the mathematical techniques to compute gravitational potential, the equations of motion can be written. These equations are defined in the body-fixed frame $(\mathrm{O} x y z)$, given in Scheeres et al. (1996), a frame with uniform rotation that follows the asteroid movement (Fig. 1). The $x y$-plane is called, in this work, rotating plane.

$\ddot{x}-2 \omega \dot{y}=\omega^{2} x+U_{x}$,

$\ddot{y}+2 \omega \dot{x}=\omega^{2} y+U_{y}, \quad$ and

$\ddot{z}=U_{z}$, 


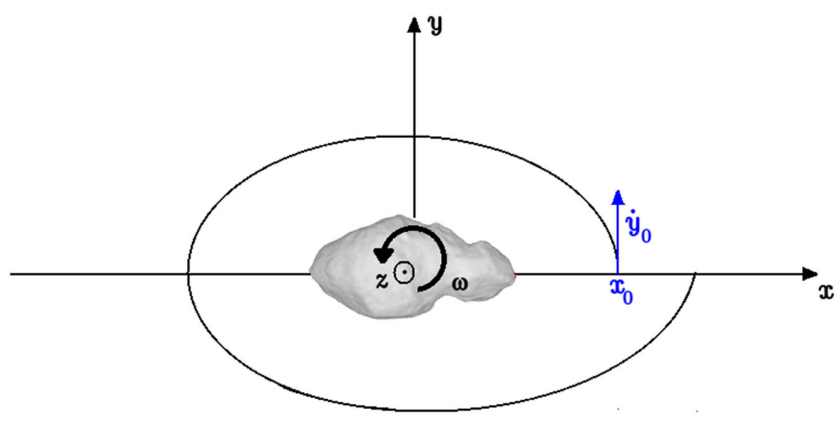

Figure 1. Schematic diagram of an orbit around a body fixed at a rotating frame. $x_{0}$ marks the initial position and the blue arrow indicates the initial velocity.

where $U_{x}, U_{y}$, and $U_{z}$ stands for the partial derivatives of the gravity potential and $\omega$ is the spin velocity of the asteroid. As mentioned before, the mascons model is used here to compute the gravitational potential, given by

$U=U(x, y, z)=-\sum_{i=0}^{N} \frac{G m}{r_{i}^{2}}$,

where $G$ is the gravitational constant, $N$ is the total number of mascons, $r_{i}$ is the distance from a mascon to the orbiting particle, and $m$ is the mass of each mascon, $m=\frac{M}{N}$ with $M$ being the total mass of the body.

Conserved quantities, such as the Jacobi constant $\left(C_{j}\right)$, can be useful to analyse the equations of motion. This constant is explicitly computed as (Scheeres et al. 1996) follows:

$C_{j}=\omega^{2}\left(x^{2}+y^{2}\right)+2 U(x, y, z)-\dot{x}^{2}-\dot{y}^{2}-\dot{z}^{2}$.

The equations (1-3) describe the motion of a massless particle and are numerically integrate via the Burlish-Stoer integrator (Bulirsch $\&$ Stoer 1966). The Poincaré section is set in the plane $y=0$ and the initial conditions are systematically distributed over the $x$-axis. It is defined $y_{0}=z_{0}=\dot{x}_{0}=\dot{z}_{0} \equiv 0, \dot{y}_{0}>0$ and $\dot{y}_{0}$ was computed for a fixed value of $C_{j}$ (equation 5). During the integration, the conditions of the orbit are saved at every instant of time when the trajectory crosses the section defined by $y=0$ with $\dot{y}>0$. The Newton-Raphson method is used to obtain an error of $\left(10^{-13}\right)$ from that section. The recorded points are plotted on the phase plane $(x, \dot{x})$ creating the Poincaré surface of section.

The method used in this work was built as a planar 2D case, adding the third dimension $(z)$ to the particle trajectory. The velocity $\dot{z}$ was set at zero in the initial condition, and during the integration, both the coordinate and the velocity are free to evolve. In order to analyse the results, the movement projected over the rotating plane was thought to be uncoupled from the movement on the third dimension. Then, the influence of $z$ variation of the orbit behaviour on the rotating plane was studied. In the Poincaré surface of section, the most internal orbit of a cluster of stability islands is called, in this paper, central orbit. Each central orbit is represented by one isolated point in the Poincaré surface of section. These points correspond to the periodic orbits in the projection over the rotating plane. Thus, the third dimension varies avoiding the repeatability, as it usually occurs in periodic orbits.

Hereinafter, we present a study about the dynamics around the asteroid 4179 Toutatis, which was chosen due to its low spin velocity. Among the asteroids with necessary data for the mascons approach, the 4179 toutatis has a rotation period of the order of days, whereas most of the other asteroids have it in hours. This period enhances the influence of the irregularity of the gravitational potential over a particle orbit. The asteroid 4179 Toutatis is an NEA and has already had several close encounters with Earth, which provided accurate data on the asteroid. A shape model was built by Ostro et al. (1995) through radar data taken in December 1992. This shape model represents the asteroid 4179 Toutatis through a polyhedron with 20000 vertices and 39996 faces (Neese 2004). Its rotational period is $5.376 \mathrm{~d}\left(T_{\text {ast }}\right)$ (Ostro et al. 1995) and the density is $2.5 \mathrm{~g} \mathrm{~cm}^{-3}$ (Scheeres et al. 1998). We apply the algorithm (Section 2) to obtain the mascons, and we consider 21106 mascon points that reproduce the gravitational potential of the asteroid.

\section{RESULTS}

In our work, the regions around the asteroid 4179 Toutatis are explored considering a range of $C_{j}$ values from 1.20 to $3.0 \times$ $10^{-7} \mathrm{~km}^{2} \mathrm{~s}^{-2}$, with $0.15 \times 10^{-7} \mathrm{~km}^{2} \mathrm{~s}^{-2}$ of interval between them. The unit of $C_{j}$ as $10^{-7} \mathrm{~km}^{2} \mathrm{~s}^{-2}$ is adopted by simplicity.

Using 75 different initial conditions for each $C_{j}$, we simulate the trajectory generated by each condition. The trajectories integration are stopped after 1000 intersections between the trajectory and the plane $y=0$. Those conditions are distributed along the $x$-axis from 2.6 to $10 \mathrm{~km}$ with steps of $0.1 \mathrm{~km}$. These simulations produce the first view of the Poincare surface of section, then, if it is necessary to complete this surface of section, new conditions are simulated.

An illustrative sample of Poincare surfaces of section is shown in Fig. 2. Three Families of periodic orbits (projections of a central orbit) were selected to be a representative sample of our results to be analysed in detail. These structures are central orbits and quasiperiodic orbits that librate around the central orbits. The Families are presented in crescent order of complexity. The islands associated to Family 1 are indicated in purple, to Family 2 in orange, and to Family 3 in red.

There is a line of points with $\dot{x}=0$ in Fig. $2, C_{j}=2.10$ and 2.25. These points indicate initial conditions whose trajectories had collided with the asteroid before the first complete orbital cycle in the rotating frame. The Poincaré surface of section for $C_{j}=1.20$ shows a line of points with $\dot{x}=0$ corresponding to unstable initial conditions whose trajectories have been ejected from the frame. The ejection condition is reached when the particle is farther than $250 \mathrm{~km}$ from the center of the asteroid. The larger is the $C_{j}$ value, the smaller is the chaotic region on the Poincare surface of section.

In Jiang et al. (2016), two Poincaré surfaces of section are built in the potential of the asteroid 216 Kleopatra to show the chaotic behaviours of the orbits in large scale. This behaviour is similar to the chaotic region seen in the present Poincaré surfaces of sections, despite of the irregular body and the dynamic region being different from the present case.

The zero-velocity surfaces can be useful to understand this behaviour. These surfaces create forbidden regions when it is projected over the $x y$-plane. For $C_{j}=1.20$, the forbidden region is open (Fig. 3a). It allows large chaotic regions. On the other hand, for $C_{j}>2.003$, the forbidden region is closed (Fig. 3b). It confines the movement, increasing the stability regions. This behaviour can be seen in more details in Winter \& Murray (1994a) and Murray \& Dermott (1999).

In this section, each selected Family will be studied. The effect of non-symmetry of the gravitational potential on the structures from the Poincaré surface of section is analysed as well.

These periodic orbits were found and followed through the Poincaré surface of section for different values of Jacob constant. Note that there are other approaches for doing that, like the 

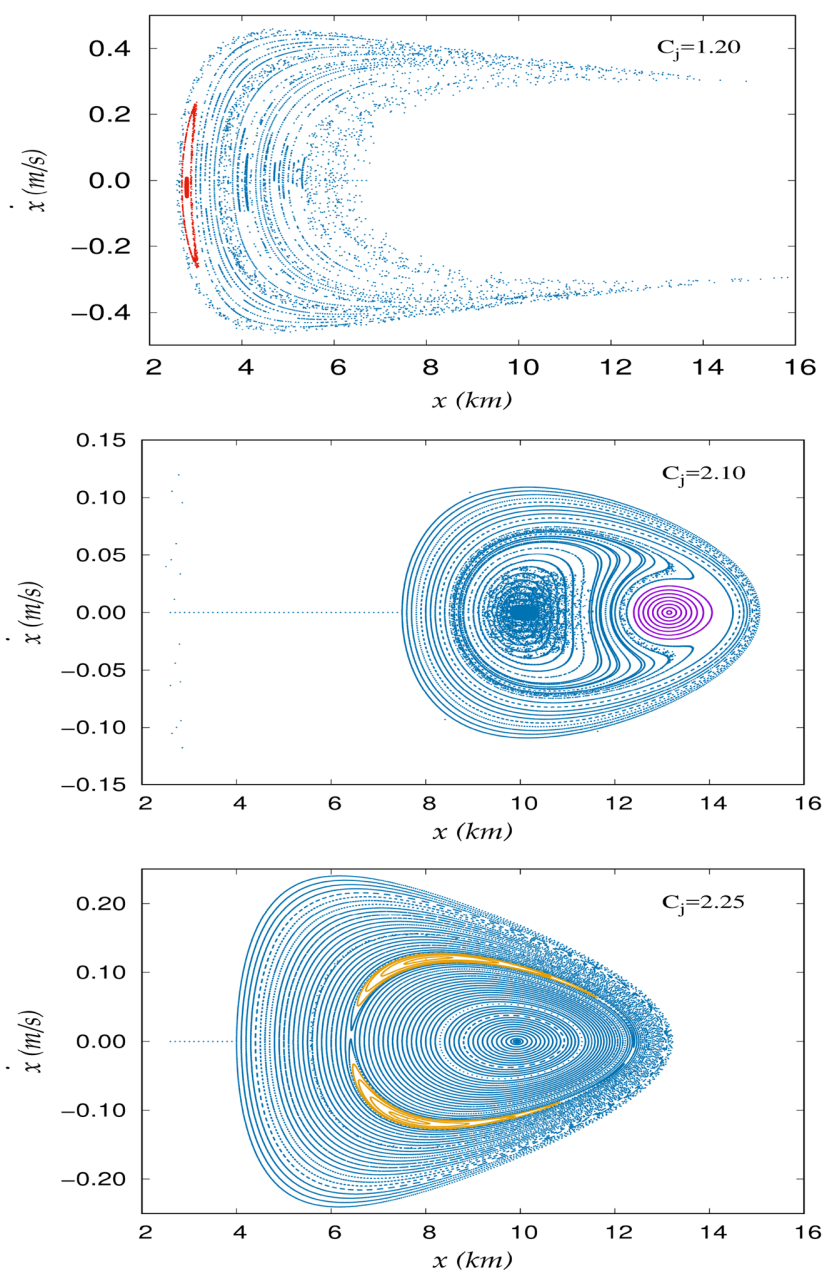

Figure 2. Poincaré surfaces of section for $C_{j}=1.20,2.10$, and 2.25. There were set initial conditions with $x_{0} \geq 2.6 \mathrm{~km}$. Up to a thousand points per initial condition are generate for each plot. The curves associated to Family 1 are indicated in purple, Family 2 are indicated in orange, and Family 3 are indicated in red.

continuation method as largely adopted in the literature (Yu, Baoyin \& Jiang 2015; Ni, Jiang \& Baoyin 2016).

\subsection{Family 1}

Family 1 is the most present one on the Poincaré surfaces of section for the studied values of $C_{j}$. Fig. 4 shows a sample of central orbits of Family 1 projected on the rotating frame and the inertial frame. These orbits are almost circular in both, the rotating frame (Fig. 4a) and the inertial frame (Fig. 4b). The central orbits are closer to the asteroid as the values of $C_{j}$ increases.

Fig. 5 shows the projections of the central orbits over the $x z$-and $y z$-planes. The variation amplitude in the $z$-axis is much smaller than the variation of the amplitude in the rotating frame. For higher values of $C_{j}$, the libration ranges of the central orbits in the $z$-axis are decreasing.

In the $3 \mathrm{D}$ rotating space, the central orbits have the shape of the contour of a hyperbolic paraboloid surface in the rotating frame (Fig. 6).

In order to illustrate the quasi-periodic orbits, in Fig. 7 is presented the libration of a large quasi-periodic orbit around a central
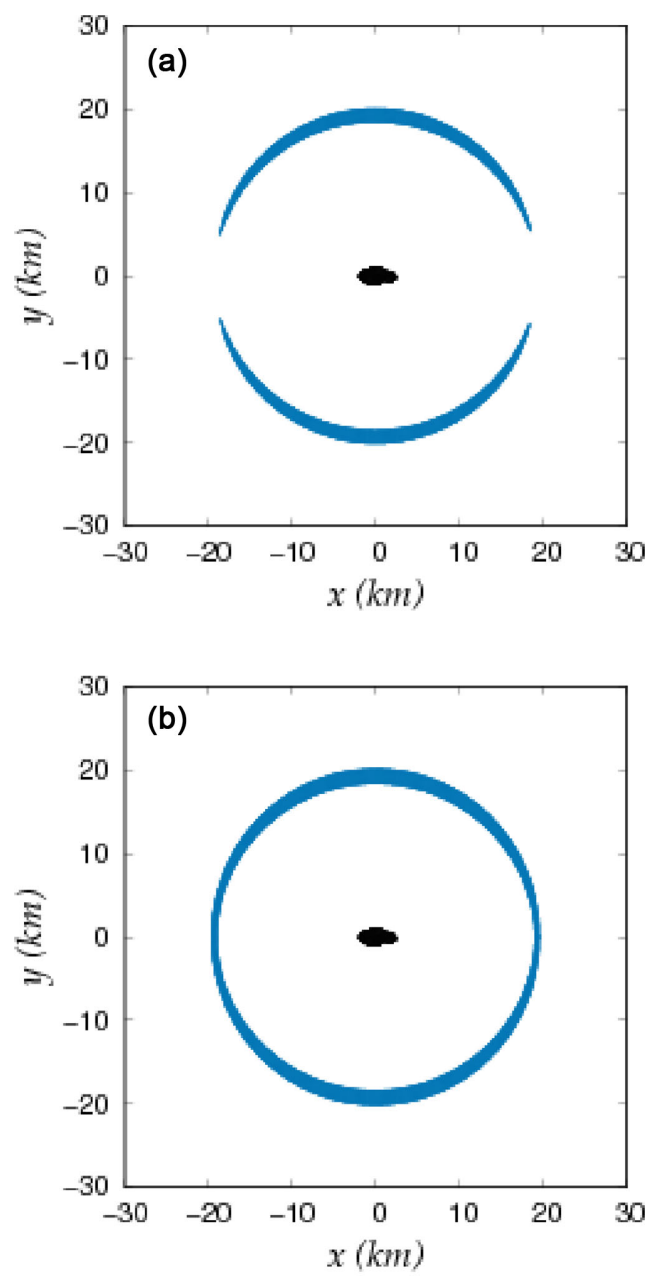

Figure 3. Illustrative cases of zero-velocity curves indicating the forbidden region (in blue). To the case (a) $C_{j}=2.003$ and the case (b) $C_{j}=2.004$.

orbit of the Family 1 with $C_{j}=2.25$. The libration conserve original structure of the central orbit. Figs 7(b) and (c) show the libration of a large quasi-periodic orbit in $x z$ - and $y z$-plane, respectively. They are asymmetric in relation to the rotating plane and the behaviour is the same for the other families studied in this work. This characteristic will be analysed in Section 4.4.

In Fig. 8, the temporal evolution of the semimajor axis, eccentricity, and inclination of a central orbit of Family 1 is presented. The semimajor axis and eccentricity have the same main frequency of variation (whose period is $\sim 0.586 T_{\text {ast }}$ ). The orbital inclination presents two main frequencies of periods $\sim 0.267 T_{\text {ast }}$ and $\sim 1.469 T_{\text {ast }}$.

The periods of the central orbits of Family 1, which are projected in the rotating plane in function of Jacobi constant, are shown in Fig. 9. The period decreases as the value of $C_{j}$ is increased. This period evolution is continuous between 0.17 and $0.58 T_{\text {ast }}$ and is not connected to a resonance, even crossing values commensurable with the asteroid period (Fig. 9). As the central orbits are not in a given resonance and the eccentricity are very low, Family 1 is classified as of the first kind (Poincaré 1899). Other classification adopted by Jiang, Yu \& Baoyin (2015a) and Jiang \& Baoyin (2016) classifies periodic orbits using the position, the geometrical, and the topological characteristics. 

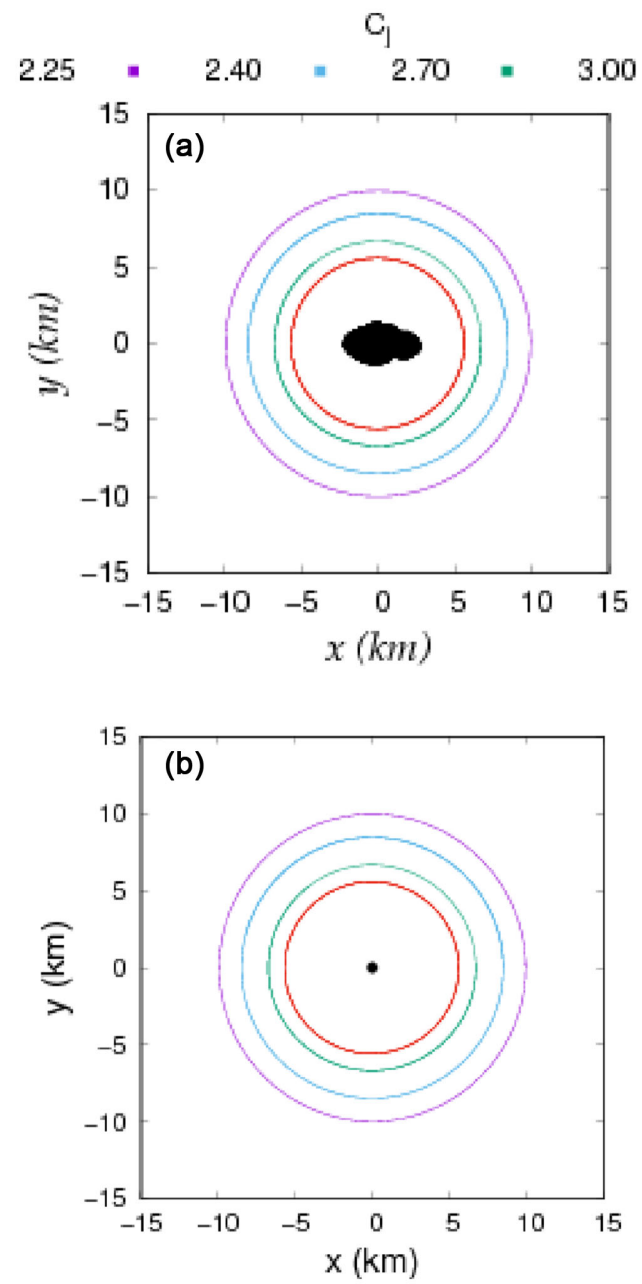

Figure 4. Sample of central orbits of Family 1: (a) in the rotating frame $(x y)$; (b) in the inertial frame (xy). The orbits completed just one cycle in the rotating frame. The colours correspond to the indicated values of $C_{j}$.

\subsection{Family 2}

Family 2 is identified by a pair of stable islands for values of $C_{j}$ from 2.19 to 2.30 . These islands are around two points in the Poincaré surfaces of section, and these two points represent the central orbit of Family 2. Fig. 10 shows the evolution of this family using the largest pair of islands, a medium pair of islands, and a pair of dots from a sample of different values of $C_{j}$. For large values of $C_{j}$, the islands come closer to each other and the amplitude of the variation( size of the islands) decreases. This behaviour is reflected in the orbital eccentricity of the trajectories. In Fig. 10, an asymmetry is identified. Such asymmetry is created by the non-symmetry of the gravitational potential. This behaviour persists even when the effect of the third dimension is neglected.

For values of $C_{j}$ lower than 2.19 and higher than 2.30, this Family does not exist. For $C_{j}<2.19$, the structure should be in a region whose trajectories collide with the asteroid.

In Fig. 11, the trajectory of the central orbit with $C_{j}=2.25$ in the rotating frame is presented. The black points are equally spaced in time and the colours indicate the modulus of the velocity in the inertial frame. The higher velocities coincide with the moment in the trajectories whose particle is closer to the asteroid, characterizing the three pericentres. On the other hand, regions with lower velocity and away from the asteroid characterize the three apocentres.
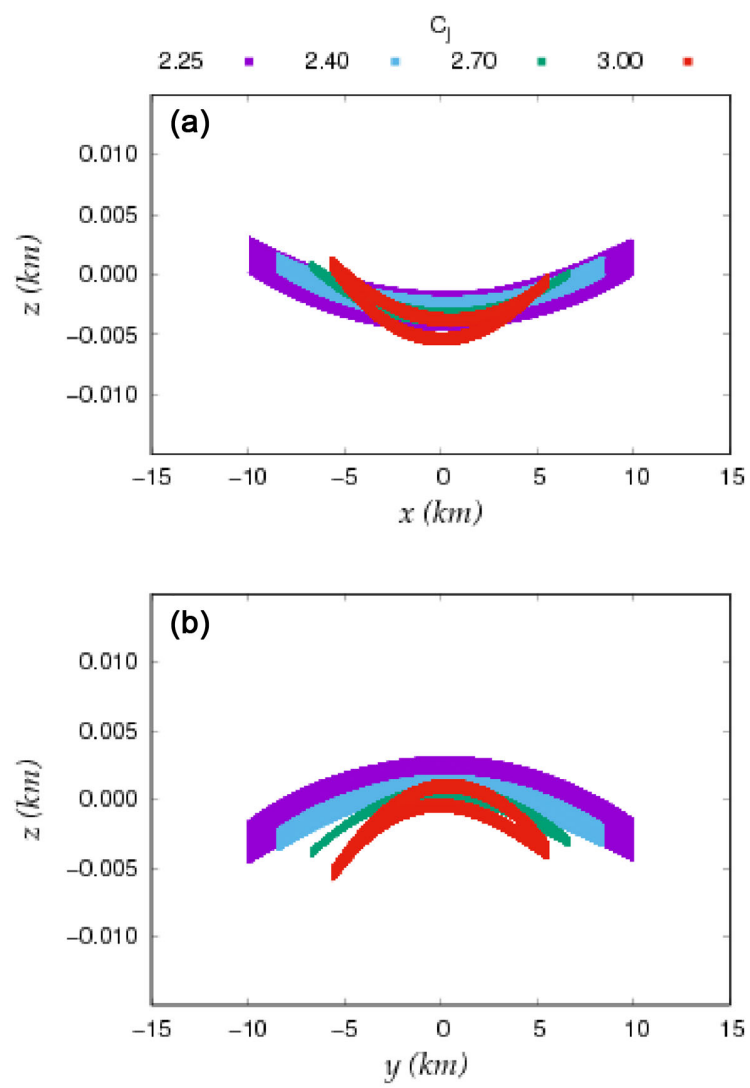

Figure 5. Sample of central orbits of Family 1: (a) projection of the trajectories in $x z$-plane; (b) projection of the trajectories in $y z$-plane, both are presented in the rotating frame. The orbits completed many cycles in the rotating frame. The colours correspond to the indicated values of $C_{j}$.

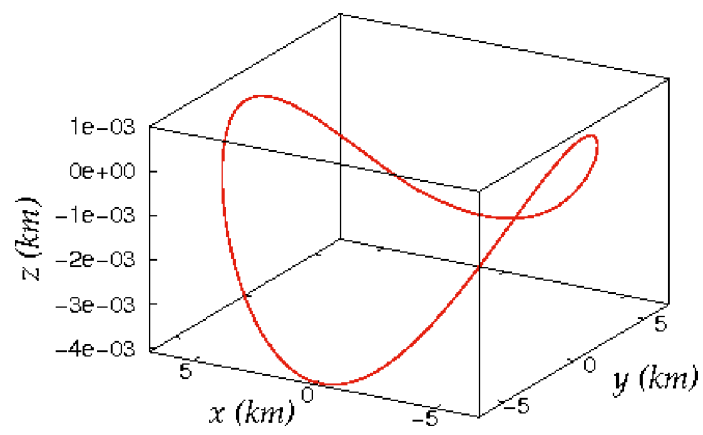

Figure 6. The central orbit with $C_{j}=2.25$. The shape is similar to the edges of a hyperbolic paraboloid. The trajectory completed many cycles in the rotating frame.

In Fig. 12, the evolution of a representative sample of central orbits in function of $C_{j}$ is presented. As far as the value of $C_{j}$ is increased, the eccentricity decreases.

In Fig. 13, the projections of central orbits in $x z$ - and $y z$-planes in the rotating frame are presented. The amplitude variation in the third dimension is much smaller than the amplitude variation in the other two dimensions. Furthermore, all variations decrease when the value of $C_{j}$ is increased.

The evolution of the semimajor axis, the eccentricity, and the inclination of the central orbit of Family 2 with $C_{j}=2.25$ are presented in Fig. 14. It is possible to identify the same main frequency (whose period is $\sim 1.028 T_{\text {ast }}$ ) in both the semimajor axis and the 

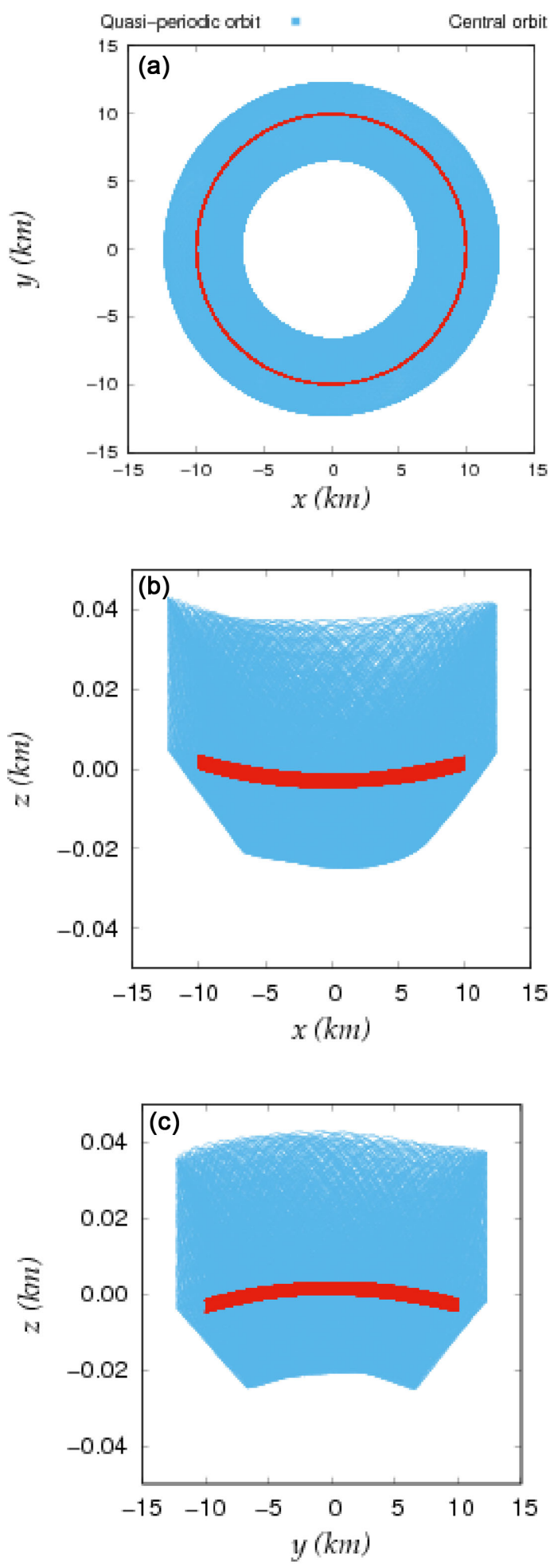

Figure 7. Projections of the central orbit (red) and a large quasi-periodic orbit (blue) of Family 1 (a) in the $x y$-plane; (b) in the $x z$-plane; (c) in the $y z$-plane. The Jacobi constant for these orbits is 2.25 .

eccentricity. The inclination has two main frequencies. The frequency with the largest period $\left(\sim 17.79 T_{\text {ast }}\right)$ is better seen in Fig. 15.

The projection of the central orbit is a periodic orbit. The period of this periodic orbit for different values of $C_{j}$ is computed, then a curve of the evolution of that period is built (Fig. 16). The periods computed are close to the rotational period of the

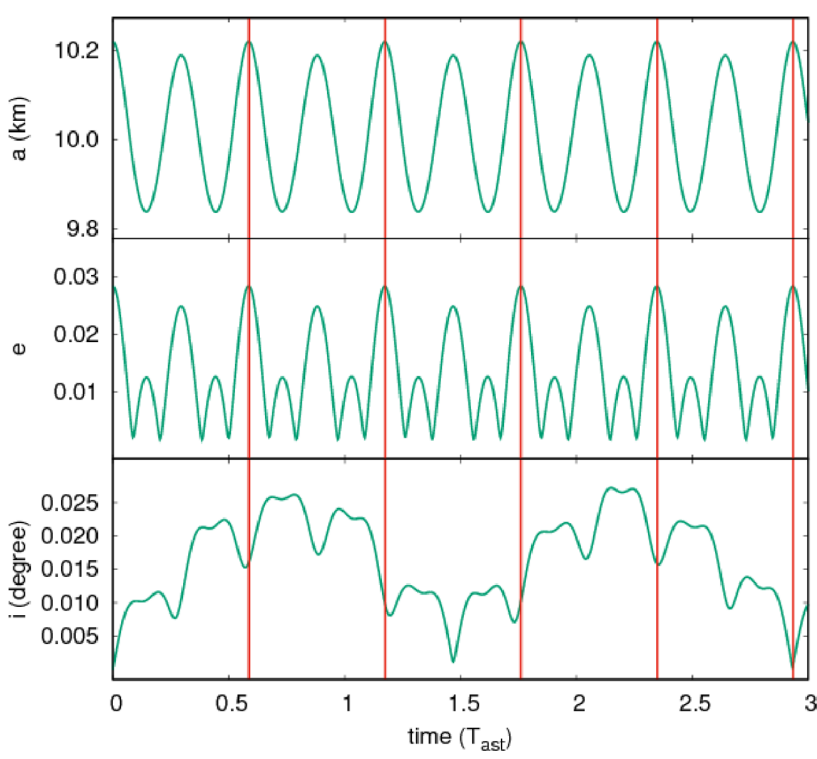

Figure 8. Temporal evolution of the semimajor axis, eccentricity and inclination of Family 1 central orbit with $C_{j}=2.25$. The red lines indicate a complete projected orbit period in the rotating frame.

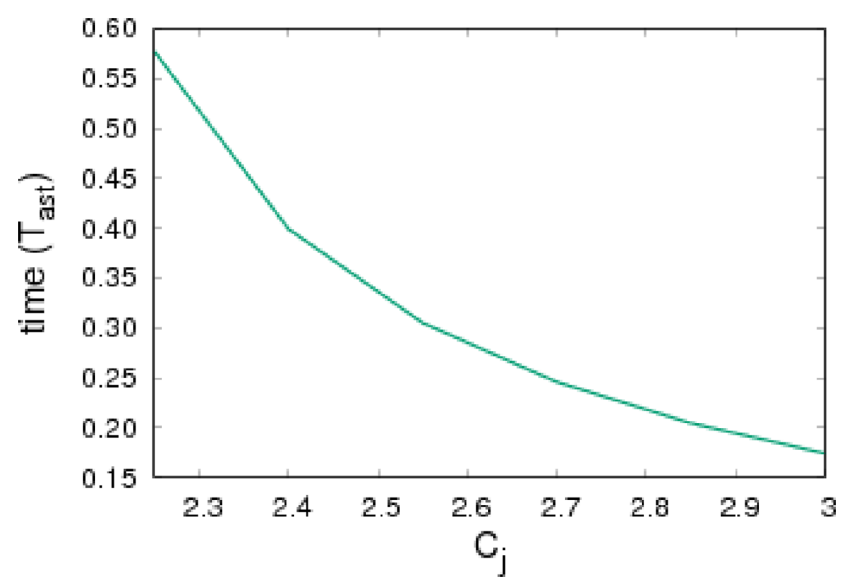

Figure 9. Evolution of the orbital period for Family 1 central orbits when projected in the rotating frame as a function of the Jacobi constant.

asteroid. In Figs 11 and 12, each complete cycle in the rotating frame corresponds approximately to three complete cycles in the inertial frame. Therefore, Family 2 is associated to the 3:1 resonance (particle orbit:asteroid rotation).

The largest quasi-periodic orbit is used to define the width of the region where the structure of the trajectories is similar to the central orbit (Fig. 17). Higher values of $C_{j}$ present central orbits with lower eccentricity and smaller libration amplitude.

Scheeres et al. (1998, see their fig. 13b) found a periodic orbit similar to a central orbit of Family 2. When using the Poincaré surface of section, we are showing that it is possible to find not only the whole Family but also the width of the libration regions.

\subsection{Family 3}

Family 3 is in the Poincaré surface of section with lower values of $C_{j}$ among the values studied here. In Fig. 18, the evolution of the family's structure on the Poincaré surfaces of section is presented with a sample of the largest island, a medium island and the point 


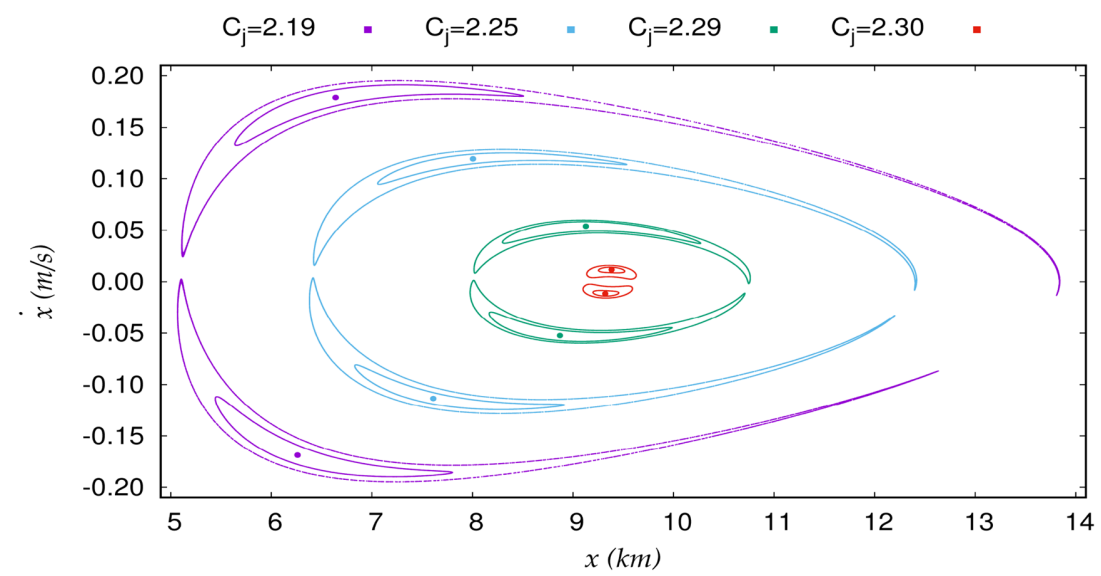

Figure 10. The evolution of Family 2 in Poincaré surfaces of section. A sample of the largest stability islands, a pair of intermediary islands and the dots inside that represent the central orbit showing the structure for different values of $C_{j}$. The colours correspond to the indicated values of $C_{j}$.

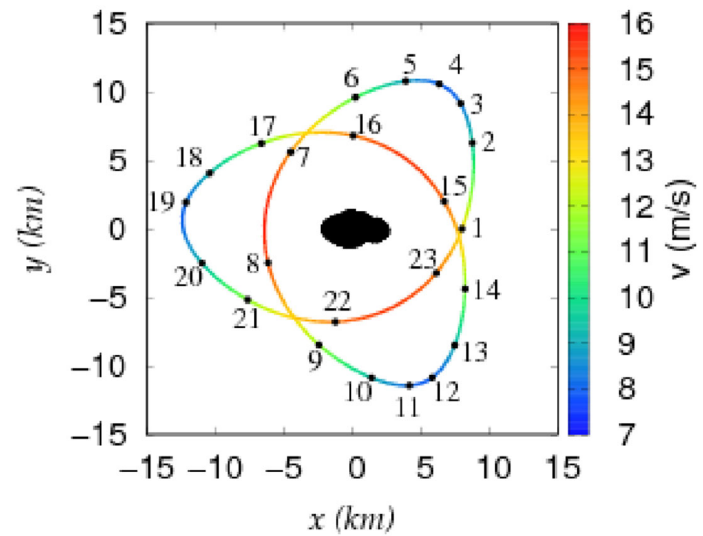

Figure 11. The central orbit of Family 2 in rotating frame for Jacobi constant 2.25. The colours indicate the velocity in the inertial frame. The orbit is divided in 23 parts with equal time steps and the points are numbered to show the trajectory sequence.

that corresponds to the central orbit. These structures with different values of $C_{j}$ were chosen in order to be a representative sample. When the value of $C j$ is increased, the width of the island becomes larger, but the island for the highest value of $C j$ (in red) breaks this behaviour. This phenomenon is due to the chaotic region where this island is placed. The chaos destroys the islands that are larger than the island in Fig. 18 with $C j=1.43$.

Fig. 19 shows the trajectory of the central orbit of Family 3 with $C_{j}=1.33$. The arrows give a sequential direction of the trajectory, and the points are plotted with equal time step. There are two regions with higher velocity and closer to the asteroid, indicating the pericentres of the orbit. The opposite situation, i.e. lower velocities and farther from the asteroid, indicates the apocentres. A difference between Family 3 and the other families is that there are moments whose trajectories become retrograde in the rotating frame. It is due to the high orbital eccentricity. The variation of the orbital velocity is wide enough to be faster than the rotating velocity of the asteroid when it is close to the pericentre and slower when it is close to the apocentre. Fig. 20 shows a sample of the central orbits in the rotating frame and in the inertial frame. It is possible to note the existence of the two pericentres and the two apocentres.

As in the other families, the amplitude of the variation in the $z$-axis is much smaller than the variation in the other axes. This
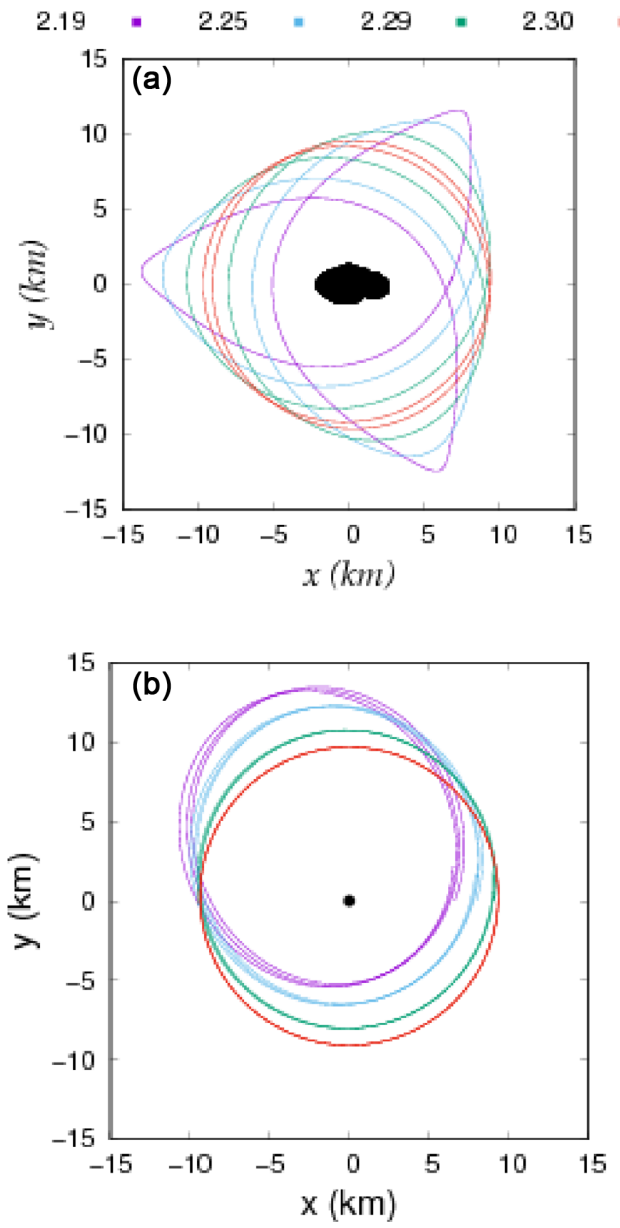

Figure 12. Central orbits from Family 2: (a) rotating frame, $x y$-plane; (b) inertial frame, $x y$-plane. The orbits completed just one cycle in the rotating frame. The colours correspond to the indicated values of $C_{j}$.

amplitude decreases when the value of $C_{j}$ is increased (Fig. 21). The variation of the structure and of the amplitude of the trajectory in $x z$ - and $y z$-planes are the smallest among the studied families. It is due to the fact that Family 3 is restricted to a small range of values of $C_{j}$. 

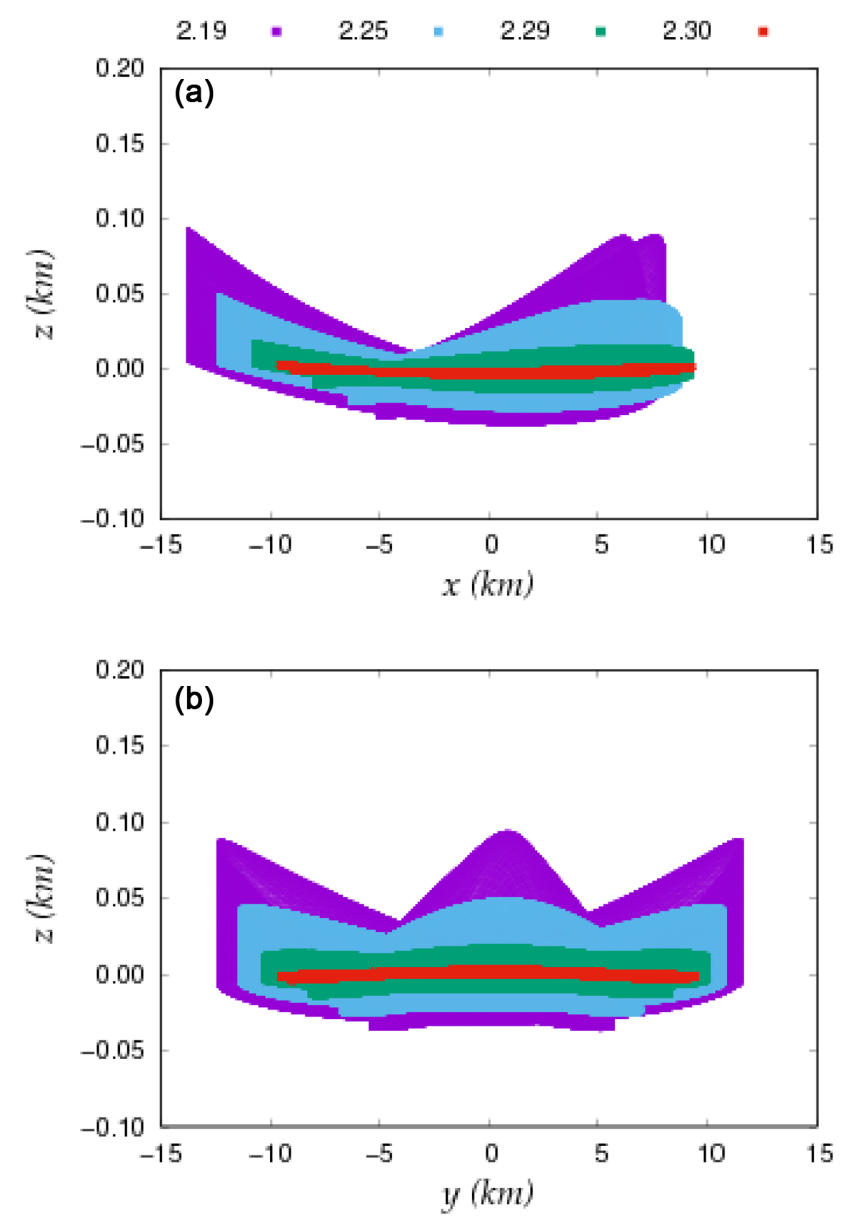

Figure 13. Central orbits from Family 2: (a) projection of the trajectories in $x z$-plane; (b) projection of the trajectories in $y z$-plane, both are presented in the rotating frame. The orbits completed many cycles at rotating frame. The colours correspond to different values of $C_{j}$.

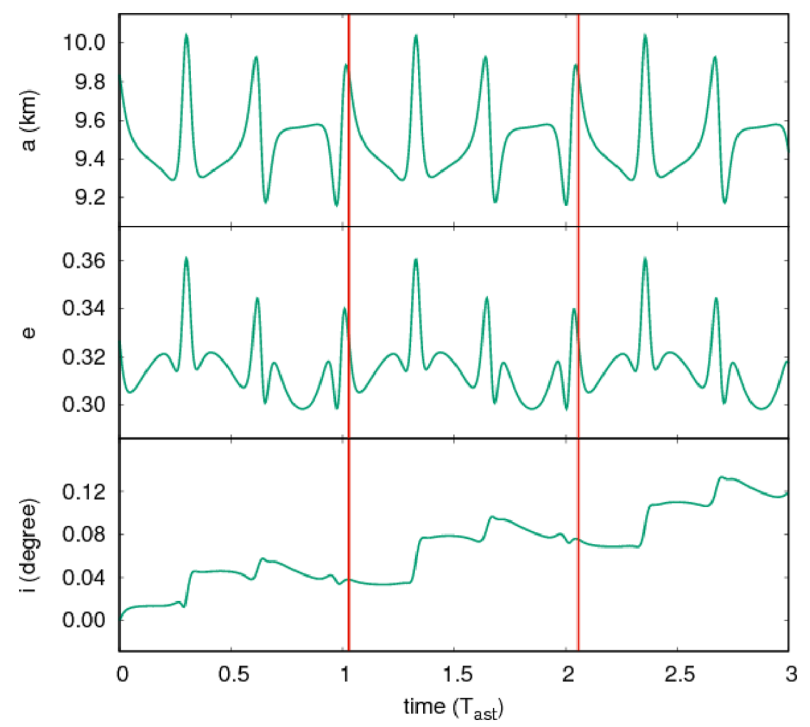

Figure 14. The evolution of the semimajor axis, eccentricity and inclination of Family 2 central orbit with $C_{j}=2.25$. The red lines correspond to a complete orbit period in the rotating frame.

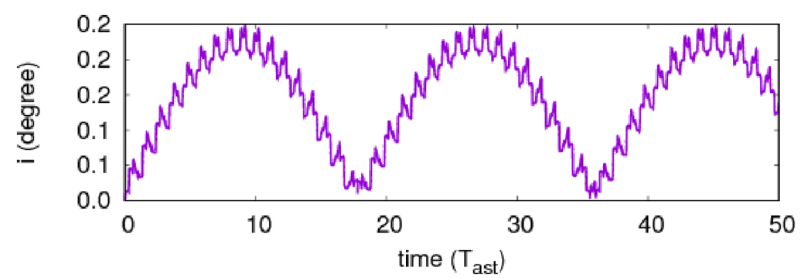

Figure 15. Evolution of the orbital inclination of Family 2 central orbit with $C_{j}=2.25$.

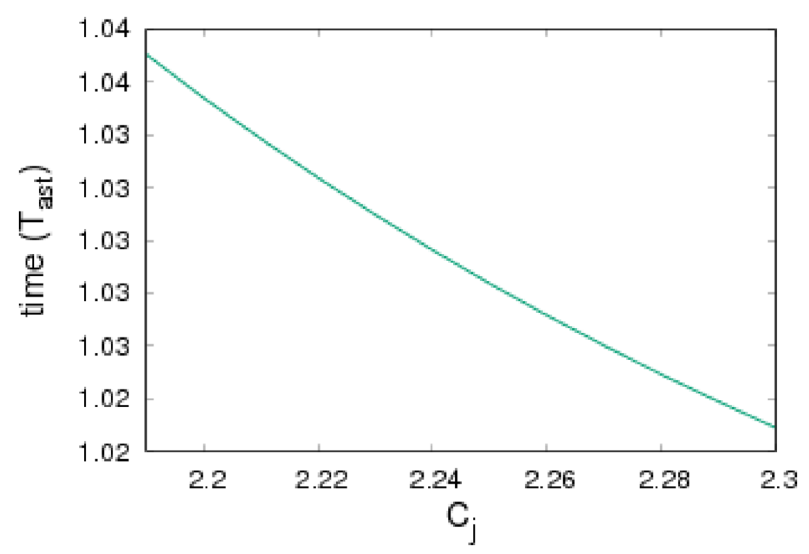

Figure 16. Evolution of the orbital period for Family 2 central orbits when projected in the rotating frame as a function of the Jacobi constant.

Fig. 22 shows the evolution of semimajor axis, eccentricity, and inclination of the central orbit with $C_{j}=1.28$. The period of the main frequency of the semimajor axis and of the eccentricity is $\sim 1.52 T_{\text {ast }}$, and the main frequency with the higher period from the inclination can be seen in Fig. 23, which is $\sim 47.07 T_{\text {ast }}$.

The period variation of the central orbits for different values of $C_{j}$ is shown in Fig. 24, where the central orbit period is close to three rotational periods of the asteroid. Given that, a cycle in the rotating frame $\left(\sim 3 T_{\text {ast }}\right)$ corresponds to two cycles in the inertial frame (Figs 19 and 20). It indicates a connection between Family 3 and the resonance 2:3(particle orbit:asteroid rotation).

A sample of the largest quasi-periodic orbits around the central orbits for different values of $C_{j}$ is presented in Fig. 25. These largest quasi-periodic orbits are the limit in which the orbits preserve the structure of the central orbit.

\subsection{The 3D effect}

Since we are dealing with a 3D system, the analysis of the Poincaré surfaces of section alone is not enough to fully understand the dynamics. In this section, we introduce an analysis of the Poincaré surfaces of section combined with the amplitude of variation of the $z$ component of the trajectories. We generated plots that show, for each initial value of $x\left(x_{0}\right)$, the upper and lower limits of the $z$ component among the points of Poincaré surfaces of section.

Thefollowing are presented: a representative sample of the Poincaré surfaces of section and their limits of variation in the third dimension. Fig. 26 shows the Poincaré surface of section for $C_{j}=3.00$ (top) and the limits of variation in the third dimension for each initial condition in the $x$-axis(bottom). For this value of the Jacobi constant, the forbidden region is closed (Fig. 3b). The initial conditions $x_{0} \geq 9.2 \mathrm{~km}$ are not integrated because they are inside the forbidden region. On the other hand, the initial conditions 

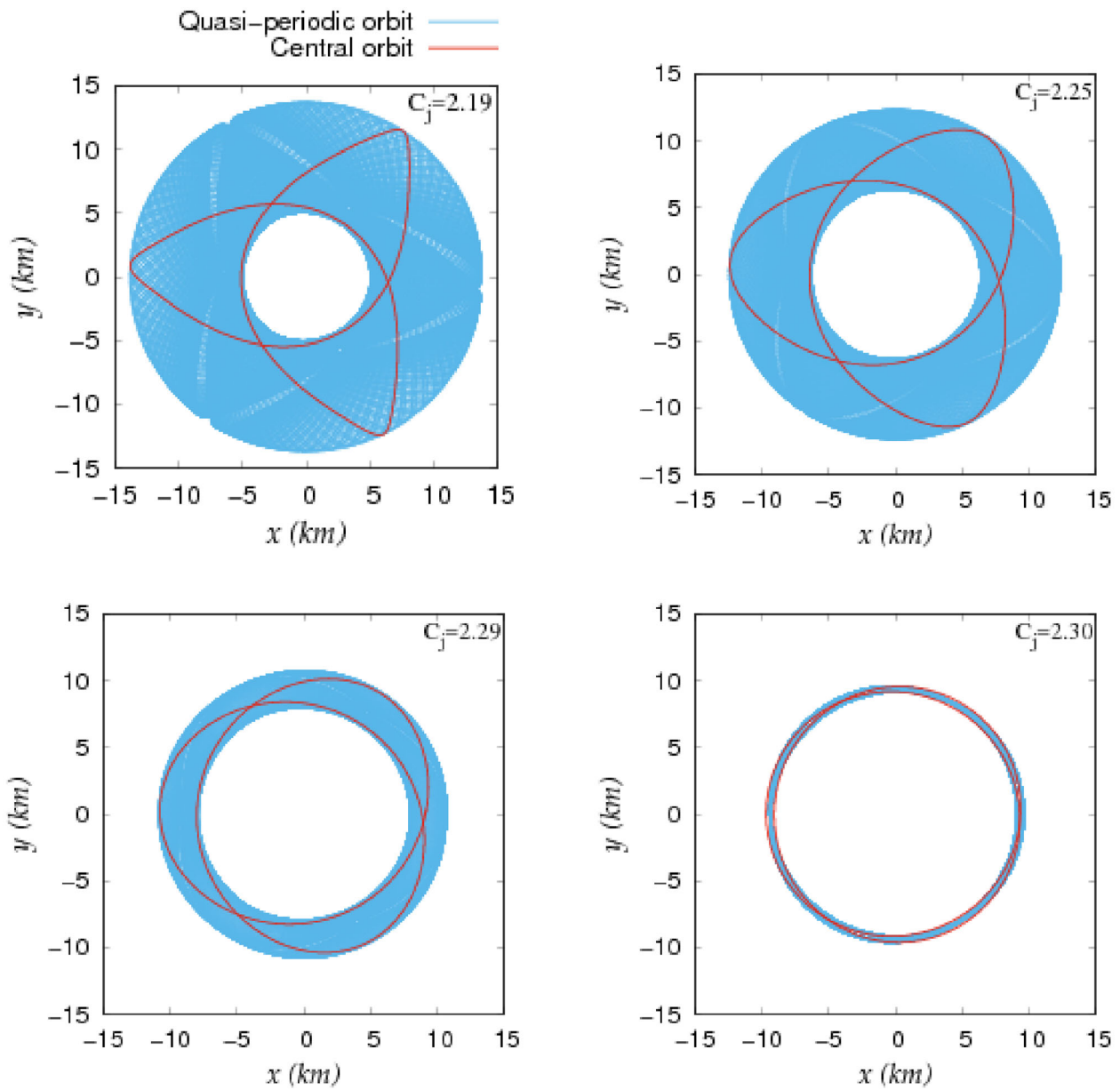

Figure 17. The largest quasi-periodic orbit (blue) and the central orbit (red) of Family 2 in the rotating frame for different values of $C_{j}$.

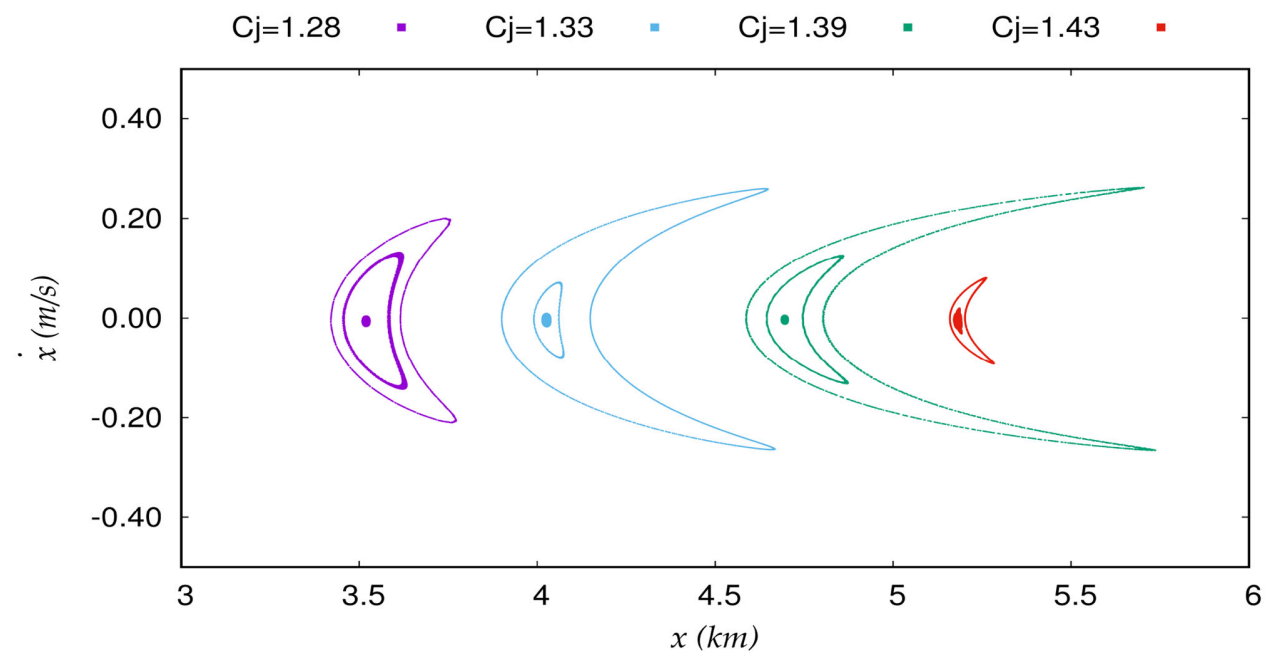

Figure 18. The evolution of Family 3 in Poincaré surfaces of section. A sample of the largest stability islands, intermediary islands and the dots that represent the central orbit showing the structure for different values of $C_{j}$. The colours correspond to the indicated values of $C_{j}$. 


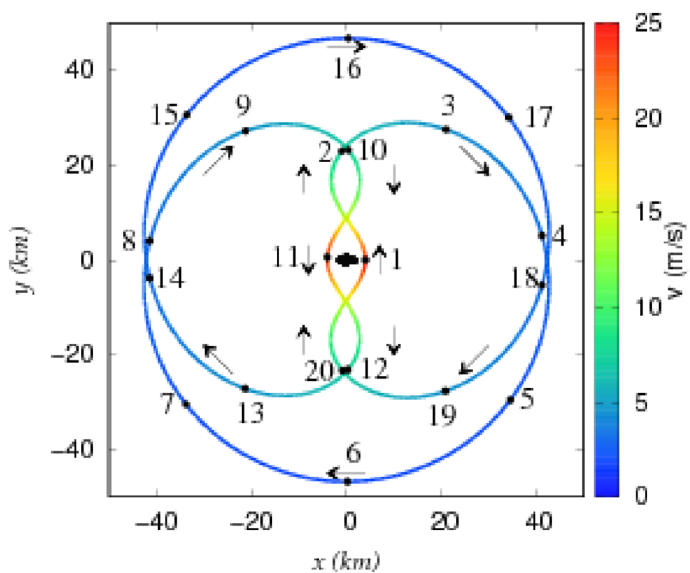

Figure 19. The central orbit from the Family 3 in rotating frame for Jacobi constant 1.33 . The colours indicate the velocity in the inertial frame. The orbit is divided in 20 parts with equal time steps, and the points are numbered to show the trajectory sequence. To assist in the understanding of the trajectory, arrows are showing the direction of the orbit.
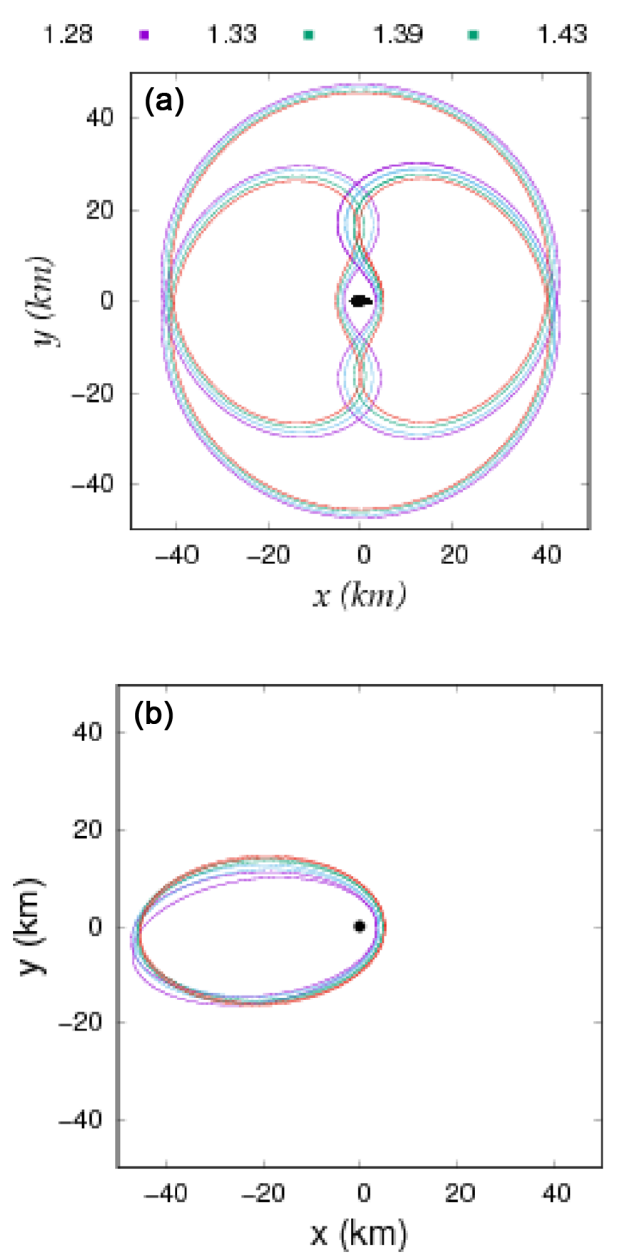

Figure 20. Central orbits from Family 3: (a) rotating frame, $x y$-plane; (b) inertial frame, $x y$-plane. The orbits completed just one cycle in the rotating frame. The colours correspond to the indicated values of $C_{j}$.
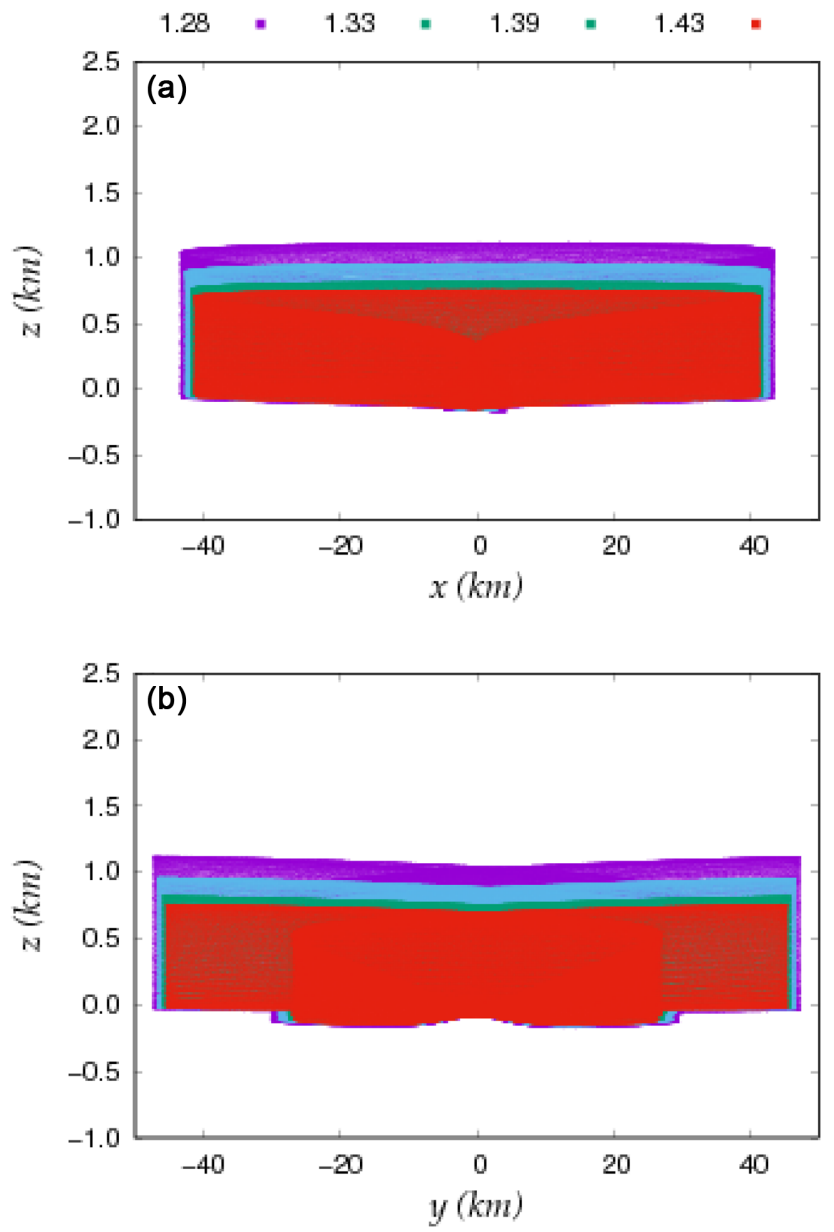

Figure 21. The central orbits from Family 3: (a) projection of the trajectories in $x z$-plane; (b) projection of the trajectories in $y z$-plane, both are presented in the rotating frame. The orbits completed many cycles at the rotating frame. The colours correspond to different values of $C_{j}$.

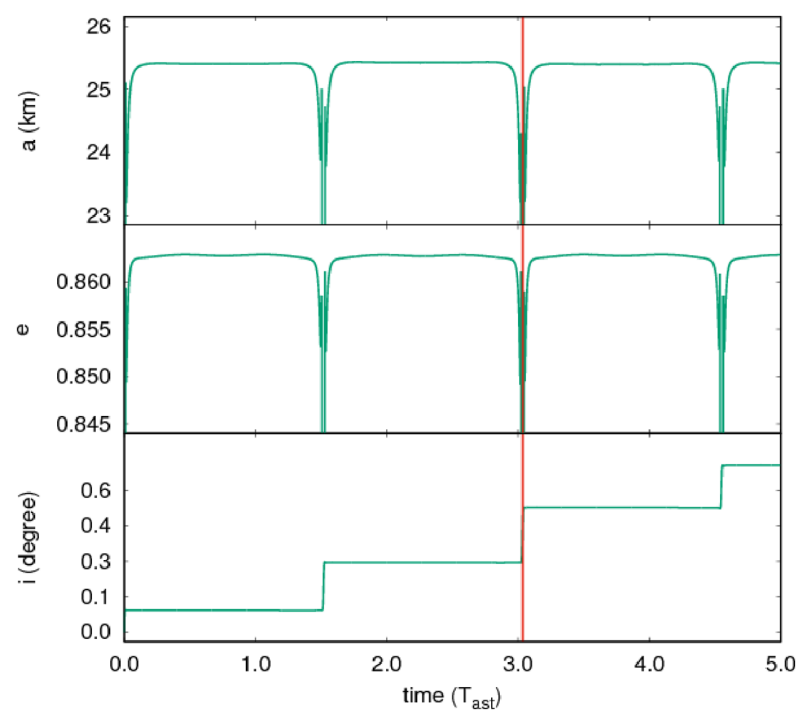

Figure 22. The evolution of the semimajor axis, eccentricity and inclination of Family 3 central orbit with $C_{j}=1.28$. The red line corresponds to a complete projected orbit period in the rotating frame. 


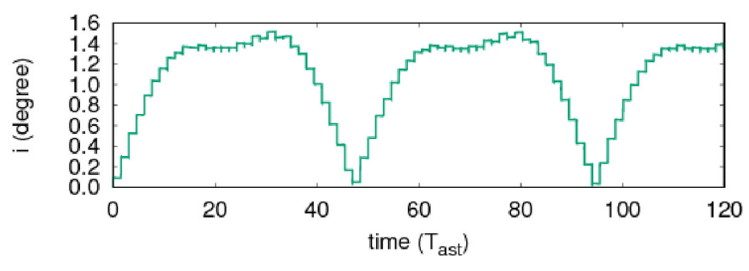

Figure 23. Evolution of the orbital inclination of Family 3 central orbit with $C_{j}=1.28$.

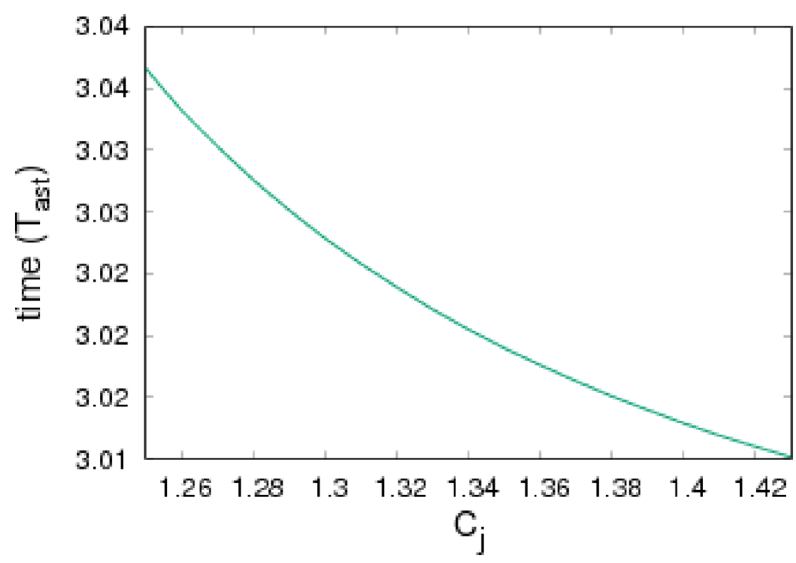

Figure 24. Evolution of the orbital period for Family 3 central orbits when projected in the rotating frame as a function of the Jacobi constant.

with $8.0 \leq x_{0}<9.2 \mathrm{~km}$ collide with the asteroid or are ejected. The limits of the variation in the third dimension are much lower than the amplitude of the trajectory in the $x y$-plane. When the initial condition is set closer to the central orbit of Family 1, the limit of variation in $z$ is closer to zero. In $x_{0}=3.7 \mathrm{~km}$, there is a break in the smoothness of the amplitude variation curve in the third dimension. In the Poincaré surface of section, this initial condition (indicated in red) does not reflect any distinct behaviour of its neighbourhood, but when we integrate the trajectory for a longer time, it became visibly chaotic.

Fig. 27 shows the Poincaré surface of section and the limits of the variation in the third dimension for $C_{j}=2.25$. For this value of $C_{j}$, the forbidden region is still closed (Fig. 3b), but smaller than the regions for higher values of $C_{j}$. In Fig. 27, the region whose initial conditions have the trajectories interrupted by the collision of the orbiting particle with the asteroid is marked in grey. Close to the central orbit, the limits in the third dimension tend to zero, as in the previous case. Some different structures are found there. In $x_{0}=5.6 \mathrm{~km}$, the points indicated in blue in the Poincaré surface of section correspond to the upper limit $\sim 1.18 \mathrm{~km}$ and the lower limit $\sim-1.10 \mathrm{~km}$ in the third dimension. These limits are outside the graph because they are much higher than the other values. Just a discontinuity is seen in the graph, which corresponds to a chaotic trajectory in the Poincaré surface of section. Therefore, the chaotic trajectory leads to a significant orbital variation in the third dimension, and vice versa. In this Poincare surface of section with the value $C_{j}=2.25$ there is a central orbit of the Family 2. In $x_{0}=6.4$ is a quasi-periodic orbit that is librating around the central orbit of Family 2, indicated in red. Note that the structure does not have a variation in the behaviour of the limit in the third dimension, since it is not chaotic.

Fig. 28 shows the Poincaré surface of section for $C_{j}=2.10$ and the respective limits of the variation in the third dimension. For this value of $C_{j}$, the forbidden region is still closed. The Poincaré surface of section presents stable and unstable regions in equal proportion. There are structures with two central orbits. Between $x_{0}=12.4$ and $14.1 \mathrm{~km}$, the limits of the $z$ variation are very close to zero compared to the others in the graph and such behaviour is similar to one in Fig. 26. This region corresponds to a stable region of quasi-periodic orbits around the central orbit of Family 1. Other stable regions can be found by the evolution of the limits in the third dimension and correspond to quasi-periodic orbits. The quasi-periodic orbits are indicated in black in the Poincaré surface of section. Peaks in $x_{0}=8.5$ and $14.2 \mathrm{~km}$ correspond to chaotic trajectories in the Poincaré surface of section. The results for $x_{0}=8.5 \mathrm{~km}$ are indicated in dark blue, and the results for $x_{0}=14.2 \mathrm{~km}$ are indicated in red. From $x_{0}=12.0$ to $12.4 \mathrm{~km}$, there is an unstable region (orange) confined between two stable regions. And between $x_{0}=9.1$ and $10.9 \mathrm{~km}$, there is an unstable region with higher limits of variations in the third dimension. In this region, each initial condition is indicated in different colours (yellow, green, and blue). There is a chaotic structure confined within this region. This structure presents a continuous behaviour in the Poincaré surface of section, and in the limits of variation in the third dimension. In the Poincare surface of section, at least one trajectory crosses from the unstable region to stable region. This phenomenon is just apparent, actually due to the third dimension. In the Poincare surface of section of $2 \mathrm{D}$ systems, this does not occur. The $2 \mathrm{D}$ case accepts just one solution of $x$ and $\dot{x}$ by the Picard-Lindelöf theorem. Therefore, there is not a crossing between the two solutions for different initial conditions in a 2D case. Here, we study a 3D case projected in a $2 \mathrm{D}$ phase space. There is just one solution for $x, z, \dot{x}$, and $\dot{z}$, but infinite number of solutions for $x$ and $\dot{x}$ with different values of $z$ and $\dot{z}$.

In Fig. 29, the Poincaré surface of section for $C_{j}=1.80$ and the respective limits of the movement in third dimension are presented. For this value of $C_{j}$, the forbidden region is opened (Fig. 3b). The stable trajectories are indicated in black. The unstable trajectories are indicated in different colours in a range from red to blue distributed from the lowest to highest values of $x_{0}$. The unstable region for this value of $C_{j}$ is larger than the unstable regions of higher values of $C_{j}$. From the limits of the variation in the third dimension, it is possible to identify the unstable and the stable regions that are clearly separated. The stable region starts from $x_{0}=4.7 \mathrm{~km}$, where the limits of the variation in the third dimension are much lower than in the region before $x_{0}=4.7 \mathrm{~km}$. The red squares indicate the initial conditions whose particles have collided with the asteroid before the trajectory completes 1000 points in the Poincaré surface of section. Comparing the section and the $z$ limits of the variation, it is possible to find chaotic trajectories that produce points in the Poincaré surface of section over the stable region, in the same way that it happened in the Poincaré surface of section for $C_{j}=2.10$ (Fig. 28).

In Fig. 30, the Poincaré surface of section for $C_{j}=1.20$ and its respective limits of variation in the third dimension are presented. For this value of $C_{j}$, the forbidden region is widely opened. In the variation within $z$ limits, there is no separation between stable and unstable regions but a mix of both. For values higher than $x_{0}=6.7 \mathrm{~km}$, all trajectories are ejected. The red squares indicate the initial conditions of these ejections.

\section{FINAL COMMENTS}

We have shown that the Poincaré surface of section technique is applicable to a three-dimension dynamical system of a particle 

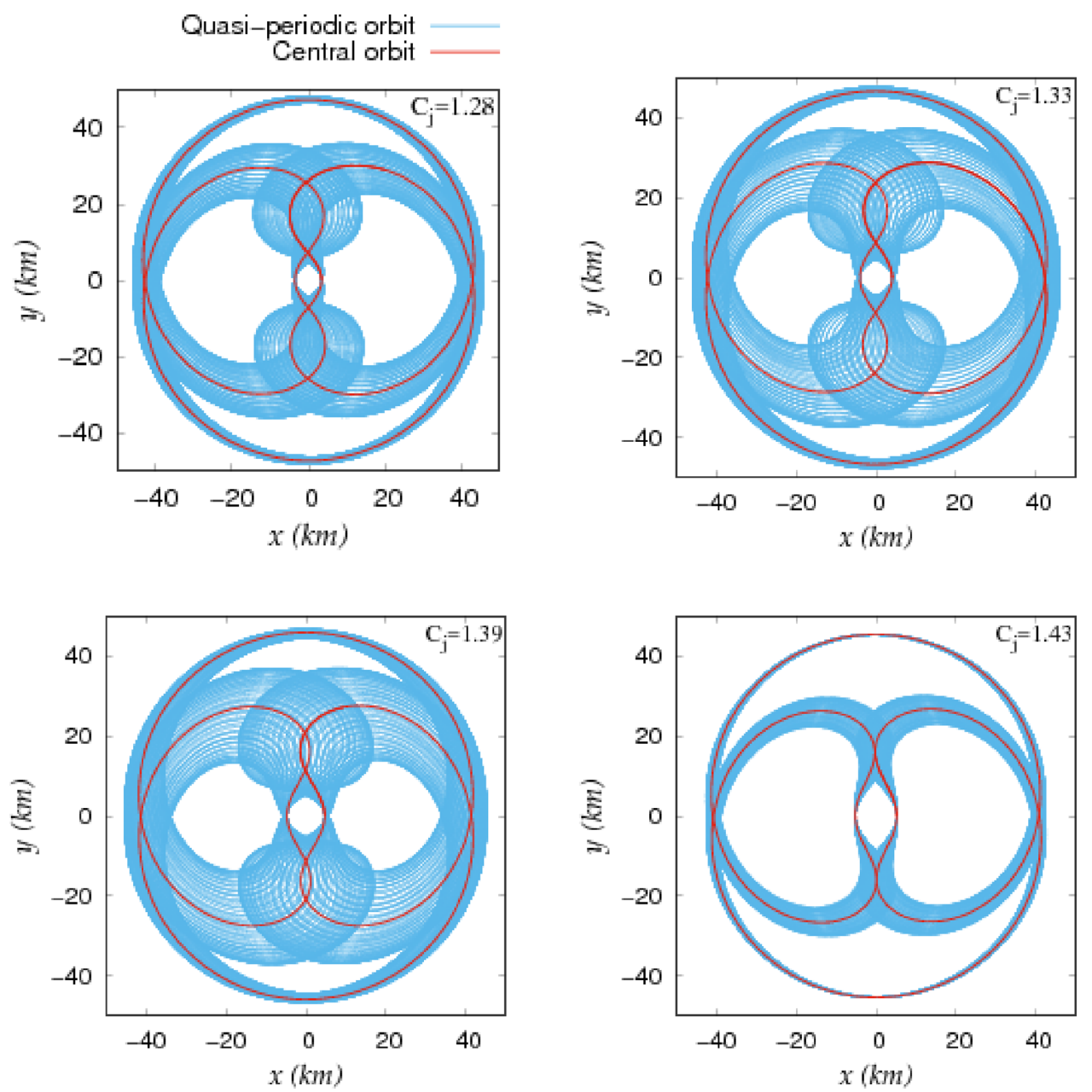

Figure 25. The largest quasi-periodic orbit (blue) and the central orbit (red) of Family 3 in the rotating frame for different values of $C_{j}$.

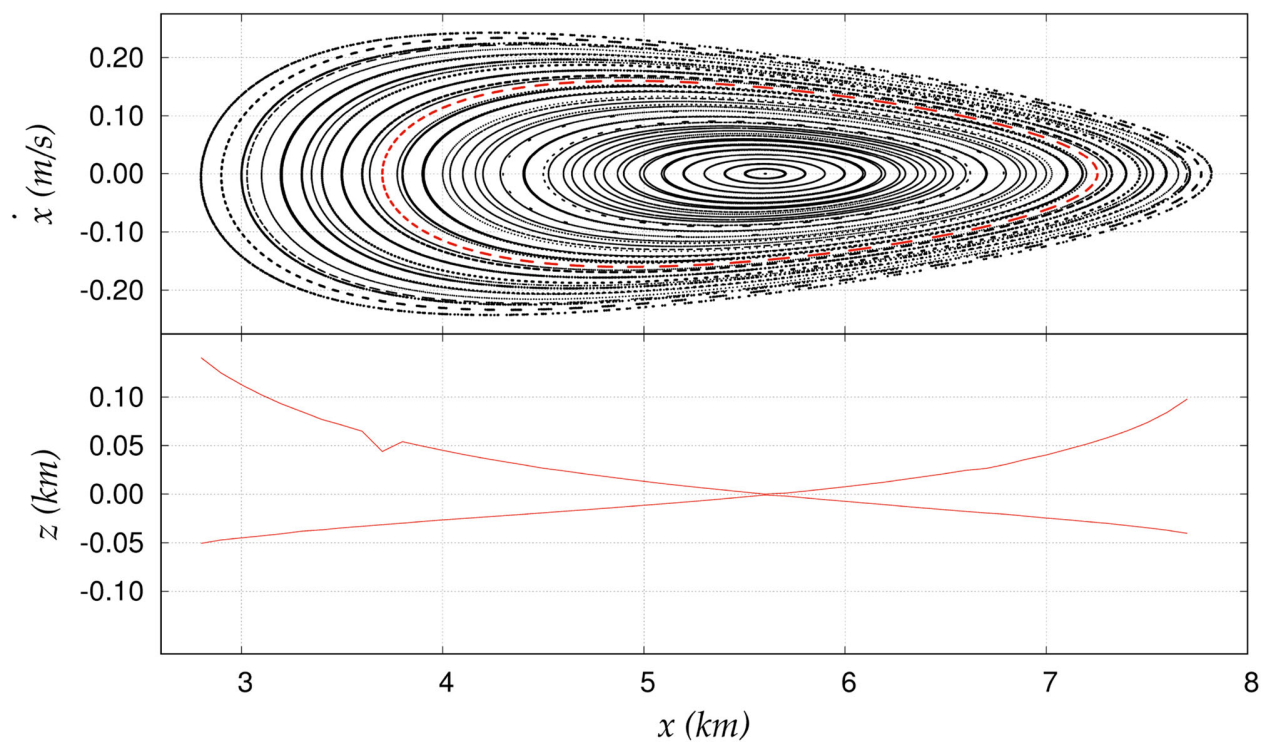

Figure 26. Poincaré surface of section for $C_{j}=3.00$ and the limits of the variation in the $z$-axis for each initial condition. A cluster of stability islands for just one initial condition is indicated in red in the surface of section. 


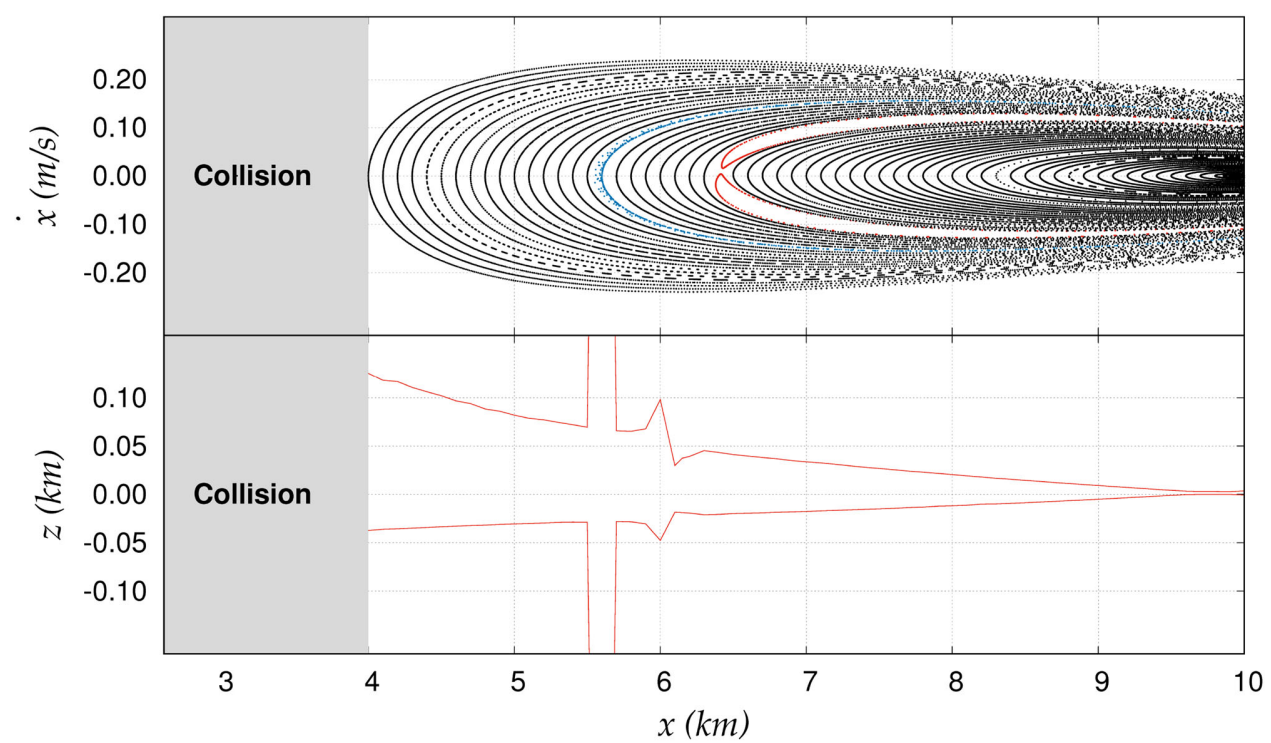

Figure 27. Poincaré surface of section for $C_{j}=2.25$ and the limits of the variation in the $z$-axis for each initial condition. A chaotic trajectory is indicated in blue in the surface of section and a pair of islands in red.

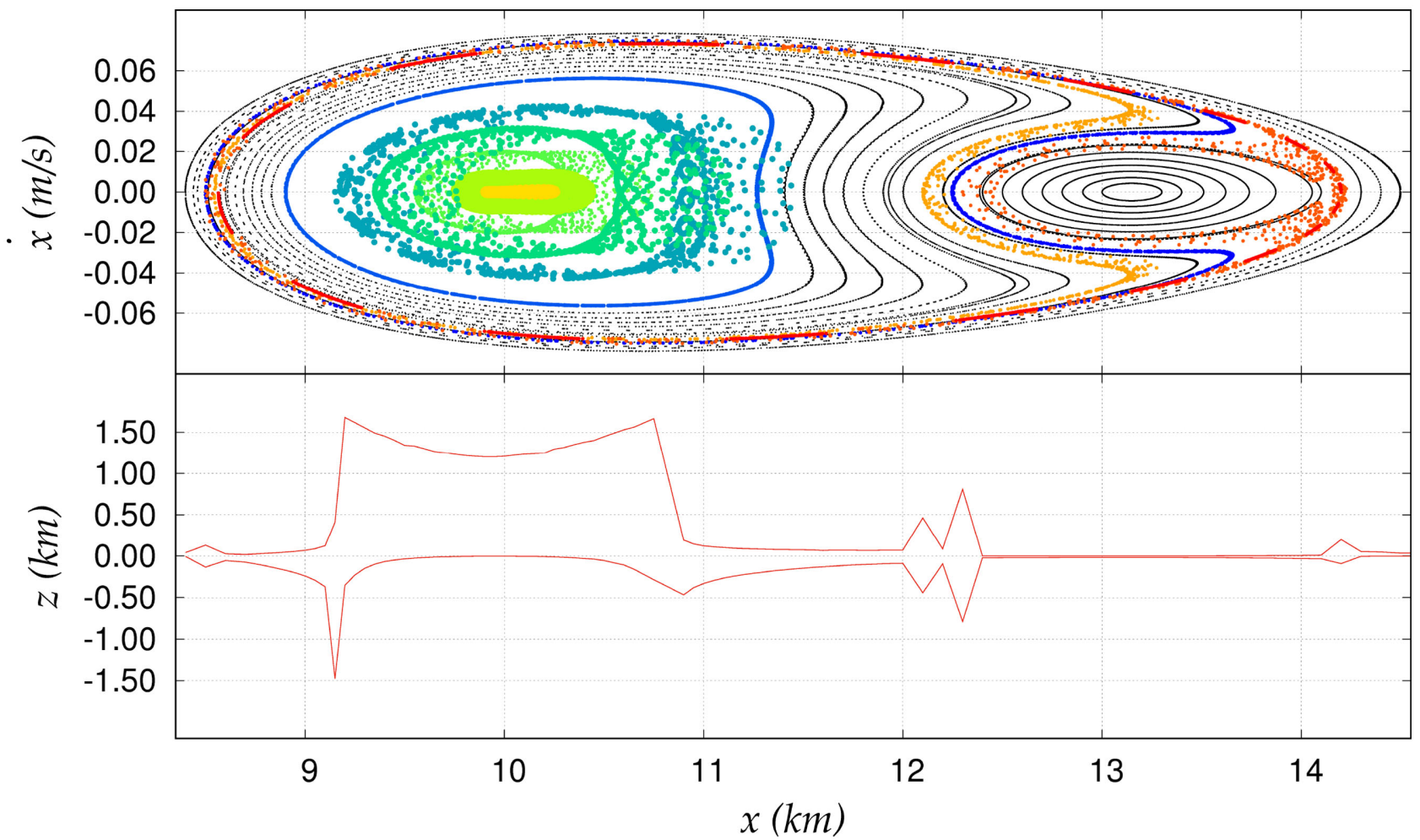

Figure 28. Poincaré surface of section for $C_{j}=2.10$ and the limits of the variation in the $z$-axis for each initial condition. The chaotic structures are indicated in different colours for each initial condition.

orbiting an irregular body whose gravitational potential is asymmetric. We applied this technique for the asteroid 4179 Toutatis, in order to study stable and unstable regions close to the asteroid. Three families of periodic orbits (projections of central orbits in the rotating plane) were selected for a detailed analyzes. The first one is a periodic orbits family of first kind, with a very low eccentricity. The other families are of second kind. The second one is a family associated to the resonance 3:1. The last one is a family associated to the resonance $2: 3$. These three families are a sample of the similarity between a usual two-dimension systems and our approach in the three-dimension case.

In the evolution of the three periodic orbit families considered, we did not identify any kind of bifurcation as those found in the results of Ni et al. (2016).

We analyse the behaviour of a representative set of Poincaré surfaces of section combined with the variation in the third dimension. Different structures than the structures of usual two-dimension systems were pointed out in the Poincaré surface of section. We found 


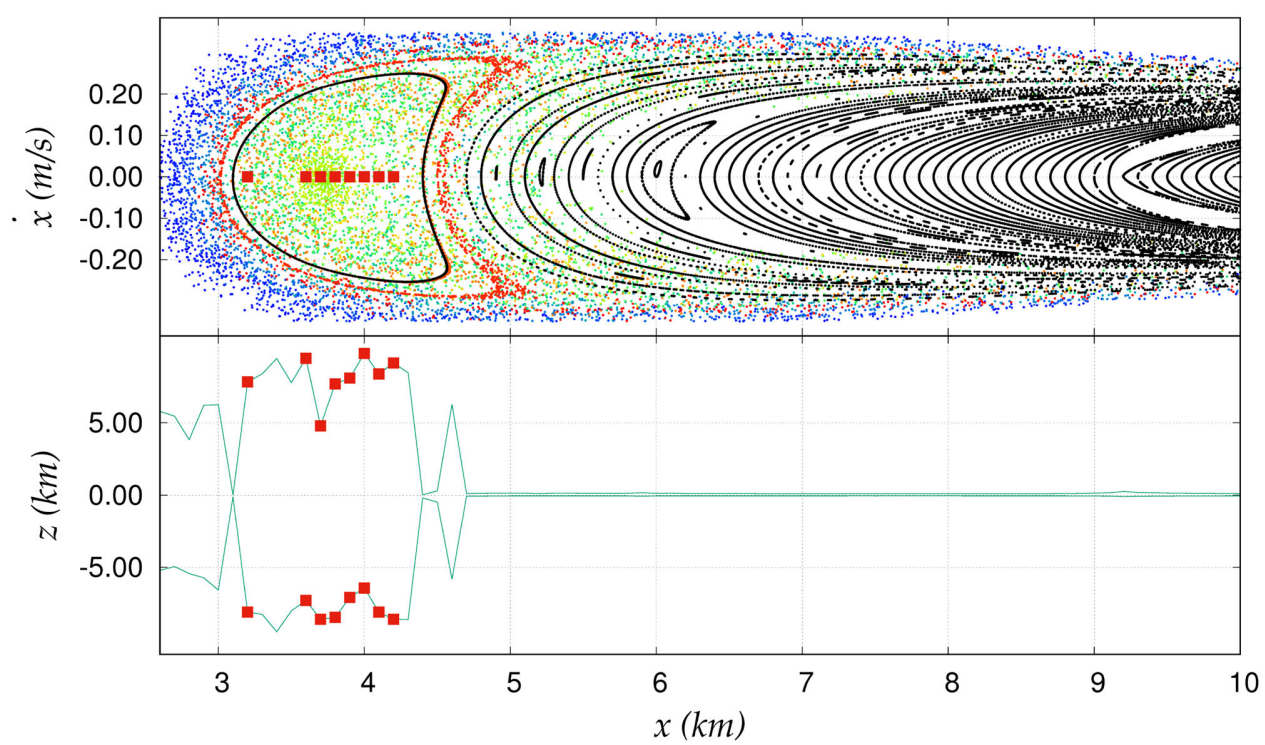

Figure 29. Poincare surface of section for $C_{j}=1.80$ and the limits of the variation in the $z$-axis for each initial condition. The chaotic structures are indicated in different colours for each initial condition. Trajectories that collided with the asteroid are indicated by the red squares.

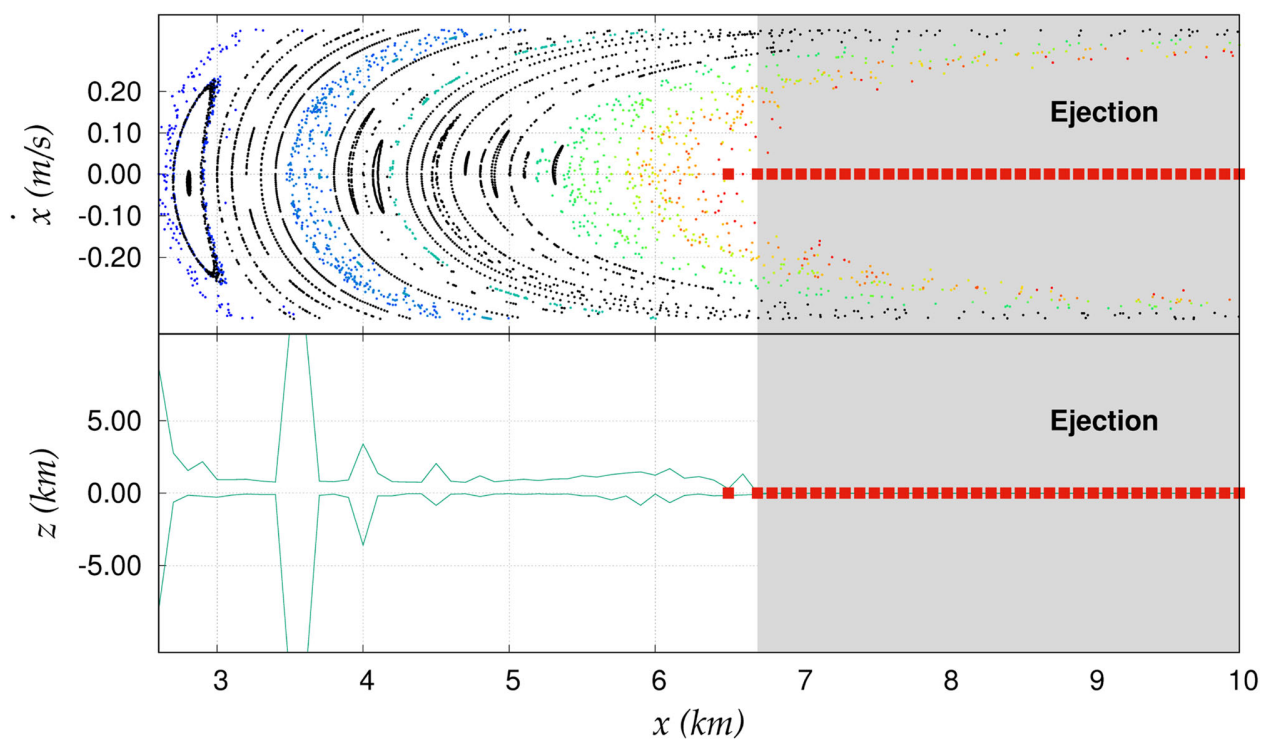

Figure 30. Poincare surface of section for $C_{j}=1.20$ and the limits of the variation in the $z$-axis for each initial condition. The chaotic structures are indicated in different colours for each initial condition. Trajectories that ejected from the system are indicated by the red squares.

a correlation between the section and the variation in the third dimension. Together with the variation in the third dimension, it is possible to identify stable and unstable regions. It is also possible to identify central orbits and libration regions around these orbits.

Despite of the three-dimension irregularity of the gravitational potential considered, we have shown that the Poincare surface of section allows the identification and location of stable and unstable regions, similarly to what has been done adopting other approaches (Frouard \& Compère 2012; Araujo et al. 2012; Jiang et al. 2015b).

\section{ACKNOWLEDGEMENTS}

We would like to thank our colleagues Ernesto Vieira Neto, Helton da Silva Gaspar, Luiz Augusto Guimarães Boldrin, Rafael Ribeiro de Sousa and Rodolfo José Bueno Rogerio for valuable discussions. This research was supported by the Brazilian agencies Coordenação de Aperfeiçoamento de Pessoal de Nível Superior CAPES, Conselho Nacional de Desenvolvimento Científico e Tecnológico - CNPq and Fundação de Amparo à Pesquisa do Estado de São Paulo - FAPESP (proc. 2016/24561-0).

\section{REFERENCES}

Abell P. A., Mazanek D. D., Reeves D. M., Chodas P. W., Gates M. M., Johnson L. N., Ticker R. L., 2017, 48th Conf., Lunar and Planetary Science Conference. The Woodlands, p. 2652

Araujo R. A. N., Winter O. C., Prado A. F. B. A., Sukhanov A., 2012, MNRAS, 423, 3058

Broucke R., Elipe A., 2005, Regular Chaotic Dyn., 10, 129

Bulirsch R., Stoer J., 1966, J. Numer. Math., 8, 1

Frouard J., Compère A., 2012, Icarus, 220, 149 
Geissler P., Petit J.-M., Durda D. D., Greenberg R., Bottke W., Nolan M., Moore J., 1996, Icarus, 120, 140

Hénon M., 1965a, Ann. d'Astrophysique, 28, 499

Hénon M., 1965b, Ann. d'Astrophysique, 28, 992

Hénon M., 1966a, Ann. d'Astrophysique, 1, 49

Hénon M., 1966b, Ann. d'Astrophysique, 1, 57

Hénon M., 1969, Acad. Sci. Paris C. R. Serie B Sci. Phys., 268, 223

Jefferys W., 1971, An Atlas of Surfaces of Section for the Restricted Problem of Three Bodies. Publications of the Department of Astronomy, The University of Texas at Austin, Austin, TX

Jiang Y., Baoyin H., 2016, AJ, 152, 137

Jiang Y., Yu Y., Baoyin H., 2015a, Nonlinear Dyn., 81, 119

Jiang Y., Baoyin H., Li H., 2015b, Astrophys. Space Sci., 360

Jiang Y., Baoyin H., Wang X., Yu Y., Li H., Peng C., Zhang Z., 2016, Nonlinear Dyn., 83, 231

Kawaguchi J., Fujiwara A., Uesugi T., 2008, Acta Astron., 62, 639

Kellogg O. D., 1954, Foundations of Potential Theory. Springer, Cambridge, MA

Laplace P. S., 1846, Oeuvres de Laplace Vol. 2. Imprimerie royale, Paris

Lauretta D. S. et al., 2017, Space Sci. Rev., 212, 925

Liu X., Baoyin H., Ma X., 2011, Astrophys. Space Sci., 334, 357

MacMillan W. D., 1936, Dynamics of Rigid Bodies. McGraw-Hill Book Company, Incorporated, New York

Murray C. D., Dermott S. F., 1999, Solar system Dynamics. Cambridge Univ. Press, Cambridge

Najid N.-E., Haj Elourabi E., Zegoumou M., 2011, Res. Astron. Astrophys., 11,345

Neese C., 2004, NASA Planetary Data System. PDS-NASA, 16

Ni Y., Jiang Y., Baoyin H., 2016, Astrophys. Space Sci., 361

Ostro S. J. et al., 1995, Science, 270, 80

Poincaré H., 1899, Les méthodes nouvelles de la mécanique céleste. No. v. 3 in Les méthodes nouvelles de la mécanique céleste, Gauthier-Villars et fils, Paris
Prockter L., Murchie S., Cheng A., Klimigis S., Farquhar R., Santo A., 2002, Acta Astron., 51, 491

Roll R., Witte L., Arnold W., 2016, Icarus, 280, 359

Rossi A., Marzari F., Farinella P., 1999, Earth Planets Space, 51, 1173

Scheeres D. J., Ostro S. J., Hudson R. S., Werner R. A., 1996, Icarus, 121, 67

Scheeres D. J., Ostro S. J., Hudson R. S., DeJong E. M., Suzuki S., 1998, Icarus, 132, 53

Shustov B. M., Naroenkov S. A., Efremova E. V., 2017, Solar Sys. Res., 51, 38

Silva A. A., Winter O. C., Prado A. F. B. A., 2009, Mathematical Problems in Engineering, Vol. 2009. Hindawi

Venditti F. C. F., 2013, PhD thesis, INPE - São José dos Campos - Brazil

Werner R. A., 1994, Celest. Mech. Dyn. Astron., 59, 253

Werner R. A., Scheeres D. J., 1996, Celest. Mech. Dyn. Astron., 65, 313

Winter O. C., 2000, Planet Space Sci., 48, 23

Winter O., Murray C., 1994a, QMW Maths Notes Vol. 16, Atlas of the Planar, Circular, Restricted Three-body Problem: Internal orbits. Queen Mary and Westfield College, London

Winter O., Murray C., 1994b, QMW Maths Notes Vol. 17, Atlas of the Planar, Circular, Restricted Three-body Problem: External orbits. Queen Mary and Westfield College, London

Winter O. C., Murray C. D., 1997a, A\&A, 319, 290

Winter O. C., Murray C. D., 1997b, A\&A, 328, 399

Yu Y., Baoyin H., Jiang Y., 2015, MNRAS, 453, 3270

This paper has been typeset from a $\mathrm{T}_{\mathrm{E}} \mathrm{X} / \mathrm{LT}_{\mathrm{E}} \mathrm{X}$ file prepared by the author. 